\title{
DIFFERENTIAL GEOMETRY OF TODA SYSTEMS
}

\author{
ALEXANDER V. RAZUMOV AND MIKHAIL V. SAVELIEV
}

\begin{abstract}
In the present paper we give a differential geometry formulation of the basic dynamical principle of the group-algebraic approach [18] - the grading condition - in terms of some holomorphic distributions on flag manifolds associated with the parabolic subgroups of a complex Lie group; and a derivation of the corresponding nonlinear integrable systems, and their general solutions. Moreover, the reality condition for these solutions is introduced. For the case of the simple Lie groups endowed with the canonical gradation, when the systems in question are reduced to the abelian Toda equations, we obtain the generalised Plücker representation for the pseudo-metrics specified by the Kähler metrics on the flag manifolds related to the maximal nonsemisimple parabolic subgroups; and the generalised infinitesimal Plücker formulas for the Ricci curvature tensors of these pseudo-metrics. In accordance with these formulas, the fundamental forms of the pseudo-metrics and the Ricci curvature tensors are expressed directly in terms of the abelian Toda fields, which have here the sense of Kähler potentials.
\end{abstract}

\section{INTRODUCTION}

The aim of the present paper is to describe some aspects of a differential and algebraic geometry foundation of a wide class of nonlinear integrable systems generated by a flat connection in the trivial fibre bundle $M \times G \rightarrow M$, with a complex Lie group $G$ and a two-dimensional manifold $M$ supplied with a complex structure. The principal example here is the well-known finite nonperiodic Toda systems (abelian as well as the nonabelian versions) associated with an arbitrary finite-dimensional simple Lie group, and their affine deformations resulting in the periodic Toda systems.

At present, there is the so-called group-algebraic approach [18] which proved itself very efficient for a constructive investigation of the integrability problem 
for nonlinear systems in low dimensions. In the framework of this method, the integrability criteria is related to such properties of the internal symmetry group of the equations under consideration, as its solvability and finiteness of the growth; and in turn is realised in the relevant properties of the characteristic algebra of the corresponding dynamical system [20,19], whose generators can be written, speaking in modern terms, as a $W$-algebra elements. At the same time, a differential and algebraic geometry setting of nonlinear integrable systems, which seems to be quite important, however, is not clear enough yet.

Our present paper is mainly devoted to the extrinsic geometry of integrable systems. Here we give a differential geometry formulation of the basic dynamical principle of the group-algebraic approach [18] - the grading condition, and its realisation in terms of some holomorphic distributions on flag manifolds $F_{ \pm}=G / \tilde{B}_{\mp}$, where $\tilde{B}_{\mp}$ are the parabolic subgroups of $G$ associated with a given $\mathbb{Z}$-gradation of the Lie algebra $\mathfrak{g}$ of the Lie group $G$. Such distributions have already been studied in mathematical literature as superhorizontal distributions [6]. Moreover, the consideration of the nonlinear Toda type systems given in our paper seems to be closely related to variations of the Hodge structures in the spirit of $\mathrm{Ph}$. A. Griffiths [13, 24]. Further, we consider the derivation of the related nonlinear systems. In particular, for the simple Lie groups one comes to the Toda systems, and just for them we reproduce a construction of their general solutions already known from [18], where they were obtained using purely Lie group methods. Moreover, here we introduce the reality condition for these solutions in terms of the corresponding mappings $M \rightarrow F_{ \pm}$. Finally, for the case of the complex simple Lie algebra endowed with the canonical gradation, when the systems under consideration are reduced to the equations of the abelian Toda system, we derive the generalised Plücker representation for the pseudo-metrics on $M$ specified by the Kähler metrics on the flag manifolds related to the maximal nonsemisimple parabolic subalgebras (closely related to the fundamental representations of $\mathfrak{g}$ ); and the generalised infinitesimal Plücker formulas for the Ricci curvature tensors of these pseudo-metrics. In accordance with these formulas, the fundamental forms of the pseudo-metrics on $M$ and the Ricci curvature tensors are expressed directly in terms of the abelian Toda fields, which have here the sense 
of Kähler potentials.

Note that the fact that for the case of the canonical distributions the Ricci curvature tensors are connected with the corresponding fundamental forms of the pseudo-metrics has been conjectured in [10] and then proved in [22], see also [25] for the symplectic algebra, while in a very brief form, and without any relation to integrable systems. Moreover, this generalisation of the Plücker representation and formulas has been obtained in [9] by an explicit calculation of the Kähler potentials on the corresponding manifolds associated with the fundamental representations of the classical simple Lie algebras (series $A, B$, $C, D)$ endowed with the canonical gradation. This last paper comes back to the pioneering, and also quite recent investigation $[7,8]$ of some geometrical structures behind $W$-algebra for the case of the abelian $A$-Toda system as a notion of a $W_{A}$-geometry of the complex projective target manifolds associated to this system. In fact, just the very remarkable papers $[7,8]$, together with numerous discussions and contacts with J.-L. Gervais, both illuminating and pleasant, served as a vigorous stimulus for our present study of the generalised Plücker relations.

It seems to us very believable that the relevant modification of the last part of our present paper, concerning generalised Plücker representation and formulas, can be done for a more wide class of nonlinear integrable partial differential equations, in particular for nonabelian versions of the finite nonperiodic Toda system. Moreover, all what we have said up to now, mainly concerns with the finite-dimensional manifolds associated with finite-dimensional Lie algebras, and in turn with the finite systems. There is also a number of very interesting papers where the infinite-dimensional Kähler geometry and Grassmannians associated with the group of smooth based loops on a connected compact Lie group were investigated, see e.g. [16] and references therein; and some aspects of the differential geometry of the affine Toda systems are treated, see e.g. $[4,5,3,2]$. Here the arising (flag) manifolds behave in many respects like finite-dimensional ones. At the same time, a study of the infinite-dimensional Kähler manifolds associated with infinite-dimensional Lie algebras, in particular, algebras of the diffeomorphisms groups, should deal with the notions and objects of a novel nature, see e.g. a consideration of the $W_{\infty}$-geometry 
of the continuous $A_{\infty}$-Toda system in [23]. However, the $W$-geometry of the dynamical systems related to the infinite-dimensional Lie algebras represents much more complicated problem, and we will not discuss it here at all.

Concluding the Introduction, we would like to explain why we supply our paper with the appendices containing more or less known information concerning the geometry of the complex and real Lie groups; the parabolic subalgebras of the simple Lie algebras and the associated flag manifolds; and $\mathbb{Z}$-gradations. The point is that at present many theoreticians work with the problems related to the Toda systems, while our language is mainly differential and algebraic geometry one. Since, as far as we know, it is not commonly accepted by them in a whole extent, we give there some notations and definitions to simplify reading of our paper. Moreover, some definitions used in the main body of the paper, e.g. a relation of $\mathfrak{g}$-valued 1 -forms and a connection forms, vertical automorphism group and gauge group, are figured in physical and mathematical literature in different forms and, sometimes, with different meanings; some notions are defined on a different level of generality, e.g. matrix-valued and generic 1-forms taking values in a Lie algebra; this is why we found it possible and reasonable to give some definitions which synthesize and interpolate, in a sense, between them.

\section{Zero Curvature Representation of Toda Equations}

2.1. Flat Connections. Let $M$ be a two-dimensional manifold supplied with a complex structure $J^{M}$, so that $M$ can be considered as a one-dimensional complex manifold. Let $G$ be a complex semisimple Lie group, and $\mathfrak{g}$ be the corresponding complex Lie algebra (see appendix A). Consider a $\mathfrak{g}$-valued 1form $\omega$ on $M$. Note that any such a form corresponds to a connection form of some connection in the trivial holomorphic principal fibre bundle $M \times G \rightarrow M$, so we call $\omega$ also a connection form, or simply a connection. Suppose that the form $\omega$ satisfies the condition

$$
d \omega(X, Y)+[\omega(X), \omega(Y)]=0
$$

for any vector fields $X, Y$ on $M$. In this case the corresponding connection in $M \times G \rightarrow M$ has zero curvature, in other words it is a flat connection. Hence, 
it is natural to call relation (2.1) the zero curvature condition. Using a local coordinate $z$ in $M$, one can write

$$
\omega=\omega_{-} d z+\omega_{+} d \bar{z}
$$

where $\omega_{ \pm}$are some mappings from $M$ to $\mathfrak{g}$. In what follows the superscripts and + mean for 1 -forms on $M$ the corresponding components in the expansion over the local basis formed by $d z$ and $d \bar{z}$. In terms of $\omega_{ \pm}$the zero curvature condition takes the form

$$
\partial_{z} \omega_{+}-\partial_{\bar{z}} \omega_{-}+\left[\omega_{-}, \omega_{+}\right]=0
$$

Here and below we use the notation

$$
\partial_{z} \equiv \partial / \partial z, \quad \partial_{\bar{z}}=\partial / \partial \bar{z} .
$$

Choosing a basis in $\mathfrak{g}$ and considering the components of the expansion of $\omega_{ \pm}$over this basis as fields, we can treat the zero curvature condition as a nonlinear system of partial differential equations for the fields.

Note that the group of vertical automorphisms of the fiber bundle $M \times G \rightarrow$ $M$ is parametrised by a mapping from $M$ to $G$. According to the tradition accepted in the physical literature, we call this group the group of gauge transformations. Let $\psi$ be a mapping from $M$ to $G$, and $\omega$ be a connection form; the gauge transformed connection form $\omega^{\psi}$ is given by the relation

$$
\omega^{\psi}(x)=\operatorname{Ad}\left(\psi^{-1}(p)\right) \omega(x)+\psi^{*} \theta(x)
$$

for any $p \in M$ and $x \in T_{p}^{\mathbb{C}}(M)$. The notation $\psi^{-1}$ in (2.5) means the mapping connected with the mapping $\psi$ by

$$
\psi^{-1}(p) \equiv(\psi(p))^{-1} .
$$

Relation (2.5) can be written formally as

$$
\omega^{\psi}=\operatorname{Ad}\left(\psi^{-1}\right) \circ \omega+\psi^{*} \theta,
$$

where $\circ$ means the composition of the mappings. For the case of a linear group, $\omega$ is a matrix valued 1 -form, $\psi$ is a matrix valued function, and Eq. (2.7) takes the form

$$
\omega^{\psi}=\psi^{-1} \omega \psi+\psi^{-1} d \psi
$$


The zero curvature condition is invariant with respect to the gauge transformations (2.7). In other words, if a connection $\omega$ satisfies this condition, then the connection $\omega^{\psi}$ also satisfies it. It is convenient to call the gauge transformations defined by Eq. (2.7), $G$-gauge transformations.

Suppose that the manifold $M$ is simply connected, then for any flat connection $\omega$ there exists a mapping $\varphi$ from $M$ to $G$ such that

$$
\omega=\varphi^{*} \theta
$$

where $\theta$ is the holomorphic Maurer-Cartan form of the group $G$ (see appendix A). Note that for a linear group one can write

$$
\omega=\varphi^{-1} d \varphi \text {. }
$$

From the other hand, for any mapping $\varphi$ the connection ${ }^{\varphi} \omega$ given by

$$
\varphi_{\omega}=\varphi^{*} \theta
$$

satisfies Eq. (2.1). Moreover, ${ }^{\varphi} \omega=0$ if and only if $\varphi$ is a constant mapping.

For any two mappings $\varphi, \psi: M \rightarrow G$ we define a new mapping $\varphi \psi$ by the relation

$$
\varphi \psi(p) \equiv \varphi(p) \psi(p)
$$

Proposition 2.1. For any two mappings $\varphi, \psi: M \rightarrow G$, the relation

$$
{ }^{\varphi \psi} \omega=\operatorname{Ad}\left(\psi^{-1}\right)^{\varphi} \omega+{ }^{\psi} \omega
$$

is valid.

Proof. For any $p \in M$ and $x \in T_{p}^{\mathbb{C}}(M)$ we have

$$
{ }^{\varphi \psi} \omega(x)=(\varphi \psi)^{*} \theta(x)=\theta\left((\varphi \psi)_{* p}(x)\right) .
$$

It is not difficult to show that

$$
(\varphi \psi)_{* p}=R_{\psi(p) * \varphi(p)} \circ \varphi_{* p}+L_{\varphi(p) * \psi(p)} \circ \psi_{* p} .
$$

Now using Eqs. (A.6) and (A.7), we arrive at Eq. (2.13).

Corollary 2.1. For any two mappings $\varphi, \psi: M \rightarrow G$,

$$
\left({ }^{\varphi} \omega\right)^{\psi}={ }^{\varphi \psi} \omega, \quad \varphi^{-1} \omega=-\operatorname{Ad}(\varphi)\left({ }^{\varphi} \omega\right) .
$$


Proposition 2.2. If the equality

$$
\varphi_{\omega}={ }^{\prime} \omega
$$

is valid, then $\varphi^{\prime} \varphi^{-1}$ is a constant mapping.

Proof. Performing the gauge transformation corresponding to the mapping $\varphi^{-1}$, from Eq. (2.17) we get the equality

$$
\varphi^{\prime} \varphi^{-1} \omega=0
$$

hence, $\varphi^{\prime} \varphi^{-1}$ is a constant mapping.

Therefore, in the case under consideration, any flat connection can be gauge transformed to zero. In this sense system (2.3) is trivial. From the other hand, the majority of two-dimensional integrable equations can be obtained from system (2.3) by imposing some gauge-noninvariant constraints on the connection $\omega$. Note that, in general, for the case of infinite dimensional Lie algebras and Lie groups one needs a generalisation of the scheme, see Refs. [18, 21 ], but in the present paper we restrict ourself to the finite dimensional case. Consider one of the methods to impose the conditions in question, giving, in fact, a differential-geometric formulation of the group-algebraic approach for integrating nonlinear systems in the spirit of Ref. [18].

2.2. Grading Condition. Suppose, following Ref. [18], that the Lie algebra $\mathfrak{g}$ is a $\mathbb{Z}$-graded Lie algebra, (see appendix $B$ ). The first condition we impose on the connection $\omega$ is the following. Let $\tilde{\mathfrak{b}}_{ \pm}$be the subalgebras of $\mathfrak{g}$ defined by Eq. (B.28). Require that the $(1,0)$-component of the form $\omega$ takes values in $\tilde{\mathfrak{b}}_{-}$, and its $(0,1)$-component takes values in $\tilde{\mathfrak{b}}_{+}$. We call this condition the grading condition. Any connection $\omega$ satisfying the grading condition is of the form $\varphi_{\omega} \omega$ for some mapping $\varphi: M \rightarrow G$; however not any mapping $\varphi$ leads to the connection ${ }^{\varphi} \omega$ satisfying this condition. Let us formulate requirements which should be imposed on $\varphi$ to guarantee the validity of the grading condition.

Denote by $\tilde{B}_{ \pm}$the connected subgroups of $G$ corresponding to the subalgebras $\tilde{\mathfrak{b}}_{ \pm}$. Since $\tilde{\mathfrak{b}}_{ \pm}$are parabolic subalgebras of $\mathfrak{g}$ (see appendix $\mathrm{B}$ ), the subgroups $\tilde{B}_{ \pm}$are parabolic subgroups of $G$; hence, the homogeneous spaces 
$F_{ \pm}=G / \tilde{B}_{\mp}$ are flag manifolds. Let $\pi_{ \pm}: G \rightarrow F_{ \pm}$be the canonical projections. Define the mappings $\varphi_{ \pm}: M \rightarrow F_{ \pm}$by

$$
\varphi_{ \pm}=\pi_{ \pm} \circ \varphi
$$

Theorem 2.1. The connection ${ }^{\varphi} \omega$ satisfies the grading conditions if and only if the mapping $\varphi_{-}$is holomorphic, and the mapping $\varphi_{+}$is antiholomorphic.

Proof. Suppose that the 1 -form $\left({ }^{\varphi} \omega\right)^{(0,1)}$ takes values in $\tilde{\mathfrak{b}}_{+}$. Thus, for any $x \in T_{p}^{\mathbb{C}}(M)$ we have

$$
\left(\pi_{-* e} \circ^{\varphi} \omega\left(\bar{P}_{p}^{M}(x)\right)=0\right.
$$

where the mapping $\bar{P}_{p}^{M}$ projects the tangent vector $x$ to its $(0,1)$-component. Equality (2.20) can be rewritten as

$$
\theta\left(\left(\varphi_{-* p} \circ \bar{P}_{p}^{M}\right)(x)\right)=0 .
$$

Note that, since $\theta$ is a form of the type $(1,0)$, the equality

$$
\theta(x)=0, \quad x \in T_{g}^{\mathbb{C}}(G),
$$

is valid if and only if

$$
P_{g}^{G}(x)=0
$$

(see appendix A). Hence, from Eq. (2.21) we have

$$
P_{\varphi_{-}(p)}^{G} \circ \varphi_{-* p} \circ \bar{P}_{p}^{M}=0
$$

for any point $p \in M$. The mapping $\varphi_{+* p}$ is real; thus, after the complex conjugation of Eq. (2.24), we get

$$
\bar{P}_{\varphi_{-}(p)}^{G} \circ \varphi_{-* p} \circ P_{p}^{M}=0 .
$$

It follows from Eqs. (2.24) and (2.25) that

$$
J_{\varphi_{-}(p)}^{G} \circ \varphi_{-* p}=\varphi_{-* p} \circ J_{p}^{M}
$$

and so the mapping $\varphi_{-}$is holomorphic.

Suppose now that the mapping $\varphi_{-}$is holomorphic. Reversing the arguments given above, we conclude that the form $\omega^{(0,1)}$ takes values in $\tilde{\mathfrak{b}}_{+}$.

The case of the mapping $\varphi_{+}$can be considered in the same way. 
We call a mapping $\varphi$ generating a connection satisfying the grading condition, a mapping satisfying the grading condition. Now, following again Ref. [18], perform a further specification of the grading condition. Here we confine ourselves by a special choice of such a specification leading to the Toda type systems; for a more general case see Ref. [18].

Let us require that the $(1,0)$-component of the connection $\omega$ takes values in the linear space $\mathfrak{g}_{-1} \oplus \mathfrak{g}_{0}$, and the $(0,1)$-component of it takes values in the linear space $\mathfrak{g}_{0} \oplus \mathfrak{g}_{+1}$. To reformulate the grading condition as a condition imposed on the corresponding mapping $\varphi$, we introduce some holomorphic distributions on the manifolds $F_{+}$and $F_{-}$. To this end, define subspaces $\tilde{\mathfrak{m}}_{ \pm}$ of $\mathfrak{g}$ by

$$
\tilde{\mathfrak{m}}_{+} \equiv \bigoplus_{m \leq 1} \mathfrak{g}_{m}, \quad \tilde{\mathfrak{m}}_{-} \equiv \bigoplus_{m \geq-1} \mathfrak{g}_{m}
$$

According to the convention of appendix B, in the case of the canonical gradation we use the notations without tildes. Note that in this case we can also define the subspaces $\mathfrak{m}_{ \pm}$as

$$
\mathfrak{m}_{ \pm} \equiv\left\{x \in \mathfrak{g} \mid\left[x,\left[\mathfrak{b}_{\mp}, \mathfrak{b}_{\mp}\right]\right] \in \mathfrak{b}_{\mp}\right\} .
$$

The subspace $\tilde{\mathfrak{m}}_{+}$is invariant with respect to the adjoint action of the subgroup $\tilde{B}_{-}$in $\mathfrak{g}$. Let $p \in F_{+}$, and $g$ be any element of $G$ such that $\pi_{+}(g)=p$. Define the subspace $\mathcal{M}_{+p} \subset T_{p}^{(1,0)}\left(F_{+}\right)$by

$$
\mathcal{M}_{+p} \equiv \pi_{+*}\left(\tilde{\mathfrak{m}}_{+g}\right)
$$

where

$$
\tilde{\mathfrak{m}}_{+g} \equiv L_{g *}\left(\tilde{\mathfrak{m}}_{+}\right) .
$$

The subspaces $\mathcal{M}_{+p}, p \in F_{+}$, generate a distribution on $F_{+}$which will be denoted $\mathcal{M}_{+}$. In the same way we can define an analogous distribution $\mathcal{M}_{-}$ on $F_{-}$.

Theorem 2.2. The connection ${ }^{\varphi} \omega$ satisfies the grading condition if an only if the mapping $\varphi_{-}$is holomorphic and $\varphi_{-_{*}}\left(\partial_{z p}\right) \in \mathcal{M}_{-_{-}(p)}$ for any $p \in M$, while the mapping $\varphi_{+}$is antiholomorphic and $\varphi_{+_{*}}\left(\partial_{\bar{z} p}\right) \in \mathcal{M}_{+_{+}(p)}$ for any $p \in M$.

Proof. The proof is similar to the proof of Theorem 2.1. 
It is natural in the situation, described in theorem 2.2, to say that the mapping $\varphi_{+}$is tangent to the distribution $\mathcal{M}_{+}$, while the mapping $\varphi_{-}$is tangent to the distribution $\mathcal{M}_{-}$. Note that this condition is directly related to the notion of a superhorizontal mapping [6].

It is clear that the grading condition is not invariant under the action of an arbitrary $G$-gauge transformation, but it is invariant under the action of gauge transformations (2.7) generated by the mappings taking values in the subgroup $\tilde{H}$, corresponding to the subalgebra $\tilde{\mathfrak{h}} \equiv \mathfrak{g}_{0}$. Such transformations form a subgroup of the group of $G$-gauge transformations. Below we call a gauge transformation from this subgroup an $\tilde{H}$-gauge transformation. To get a system of equations having not $\tilde{H}$-gauge invariance, we should impose further restrictions on the connection form. In fact, we choose following Ref. [18] another way leading to the system in question. It consists in constructing some $\tilde{H}$-gauge invariant quantities, and in rewriting the zero curvature conditions in terms of them. To this end, let us first consider the structure of the holomorphic principal fibre bundles $G \rightarrow F_{ \pm}$.

2.3. Modified Gauss Decomposition. Recall that the subspaces $\tilde{\mathfrak{n}}_{ \pm}$defined by Eq. (B.26) are subalgebras of $\mathfrak{g}$. Denote by $\tilde{N}_{ \pm}$the corresponding connected subgroups of $G$. Let us formulate and prove two useful lemmas.

Lemma 2.1. Let $h$ be an arbitrary element of $G$; if the set $\tilde{N}_{+} \cap h \tilde{B}_{-} \neq \emptyset$, it contains just one element of $G$.

Proof. Note that $\tilde{N}_{+} \cap \tilde{B}_{-}=\{e\}$. Suppose that $n_{1}, n_{2} \in \tilde{N}_{+} \cap g \tilde{B}_{-}$, then there exist the elements $b_{1}, b_{2} \in \tilde{B}_{-}$, such that

$$
n_{1}=h b_{1}, \quad n_{2}=h b_{2}
$$

This relation implies the equality

$$
n_{1}^{-1} n_{2}=b_{1}^{-1} b_{2}
$$

Hence, we have $n_{1}^{-1} n_{2}=e$. That was to be proved.

Lemma 2.2. The set $\pi_{+}\left(\tilde{N}_{+}\right)$is an open set. 
Proof. It is clear that $\pi_{+}\left(\tilde{N}_{+}\right)=\pi_{+}\left(\tilde{N}_{+} \tilde{B}_{-}\right)$. The subspaces $\tilde{\mathfrak{n}}_{+}$and $\tilde{\mathfrak{b}}_{-}$are such subalgebras of $\mathfrak{g}$ that

$$
\mathfrak{g}=\tilde{\mathfrak{n}}_{+} \oplus \tilde{\mathfrak{b}}_{-}
$$

hence, the mapping $(n, b) \in \tilde{N}_{+} \times \tilde{B}_{-} \rightarrow n b \in G$ is regular for any $(n, b) \in$ $\tilde{N}_{+} \times \tilde{B}_{-}$(see, for example, [15, Ch. VI]). It follows from this property that the set $\tilde{N}_{+} \tilde{B}_{-}$is open. From the other hand, the mapping $\pi_{+}$is an open mapping; thus $\pi_{+}\left(\tilde{N}_{+}\right)$is an open set.

Using the assertions of these two lemmas, we can define a local section $s_{+}$ of the fibre bundle $G \rightarrow F_{+}$, assuming that

$$
s_{+}(p) \equiv \tilde{N}_{+} \cap\left(\pi_{+}\right)^{-1}(p), \quad p \in \pi_{+}\left(\tilde{N}_{+}\right) .
$$

Since $\tilde{N}_{+}$is a holomorphic submanifold of $G$, the section $s_{+}$is holomorphic.

The subspaces $\tilde{\mathfrak{n}}_{+g} \equiv L_{g *}\left(\tilde{\mathfrak{n}}_{+}\right), g \in G$, generate an involutive holomorphic distribution $\mathcal{N}_{+}$on $G$. The image $\tilde{N}_{+}$of the section $s_{+}$is an integral manifold of this distribution. Note that for any $g \in G$ the set $g \tilde{N}_{+}$is also an integral manifold of the distribution $\mathcal{N}_{+}$. The following proposition is now almost evident.

Proposition 2.3. There exists an open covering $\left\{U_{+a}\right\}_{a \in A}$ of the manifold $F_{+}$, and a family of local holomorphic sections $\left\{s_{+a}\right\}_{a \in A}: U_{+a} \rightarrow G$ of the fibre bundle $G \rightarrow F_{+}$, such that for any $a \in A$ the set $s_{+a}\left(U_{+a}\right)$ is an integral manifold of the distribution $\mathcal{N}_{+}$.

Proof. As the first element of the required covering and the corresponding section we can take the set $U_{+}=\pi_{+}\left(\tilde{N}_{+}\right)$and the section $s_{+}$defined above. Let $p \notin U_{+}$, and $g \in\left(\pi_{+}\right)^{-1}(p)$. The set $g \tilde{N}_{+}$possesses properties similar to the properties of the set $\tilde{N}_{+}$. Namely, if $g \tilde{N}_{+} \cap h \tilde{B}_{-} \neq \emptyset$, then the set $g \tilde{N}_{+} \cap h \tilde{B}_{-}$contains just one point, and the set $\pi_{+}\left(g \tilde{N}_{+}\right)$is open. Therefore, we can define a local holomorphic section $s_{+}^{\prime}: U_{+}^{\prime} \rightarrow G$, where $U_{+}^{\prime}=\pi^{+}\left(g \tilde{N}^{+}\right)$. Here the set $U_{+}^{\prime}$ contains the point $p$. Repeating this procedure, we get a family of local holomorphic sections of the fibre bundle $G \rightarrow F^{+}$with the required properties. 
Actually we shall consider families of local sections constructed with the help of the procedure used in the proof of Proposition 2.4. In this case, for any $a \in A$ we have

$$
s_{+a}\left(U_{+a}\right)=g_{+a} \tilde{N}_{+} \equiv \tilde{N}_{+a}
$$

for some $g_{+a} \in G$. It is clear that a similar family of local sections can be constructed also for the fibre bundle $G \rightarrow F_{-}$. If the Lie algebra $\mathfrak{g}$ is endowed with an involutive antilinear automorphism consistent with the $\mathbb{Z}$-gradation, such a family of sections of the fibre bundle $G \rightarrow F_{-}$can be constructed on the base of the given family of sections of the fibre bundle $G \rightarrow F_{+}$. The corresponding method to do that is considered in section 3 .

It is known that any family of holomorphic local sections of a holomorphic principal fibre bundle, covering the base space, allows to introduce an atlas of the fibre bundle under consideration. The corresponding procedure in our case looks as follows.

Let $a \in A$, consider a holomorphic mapping $m_{+a}:\left(\pi_{+}\right)^{-1}\left(U_{+a}\right) \rightarrow G$ defined as

$$
m_{+a}(g) \equiv s_{+a}\left(\pi_{+}(g)\right), \quad g \in\left(\pi_{+}\right)^{-1}\left(U_{+a}\right) .
$$

This mapping allows to introduce another holomorphic mapping $b_{-a}$ defined on $\left(\pi_{+}\right)^{-1}\left(U_{+a}\right)$ by

$$
b_{-a}(g) \equiv\left(m_{+a}(g)\right)^{-1} g .
$$

Thus, for any $g \in\left(\pi_{+}\right)^{-1}\left(U_{+a}\right)$ we can write

$$
g=m_{+a}(g) b_{-a}(g) .
$$

Since $s_{+a}$ is a section of the fibre bundle $G \rightarrow F_{+}$, i.e.,

$$
\pi_{+} \circ s_{+a}=\mathrm{id}_{U_{+a}},
$$

we have

$$
\pi_{+}\left(m_{+a}(g)\right)=\pi_{+}(g),
$$


and from (2.38) it is readily seen that the mapping $b_{-a}$ takes values in the subgroup $\tilde{B}_{-}$. Note here that the mappings $m_{+a}$ and $b_{-a}$ have the following properties:

$$
m_{+a}(g b)=m_{+a}(g), \quad b_{-a}(g b)=b_{-a}(g) b
$$

for any $b \in B_{-}$.

It is clear that the mapping $\psi_{+a}:\left(\pi_{+}\right)^{-1}\left(U_{+a}\right) \rightarrow U_{+a} \times \tilde{B}_{-}$, defined as

$$
\psi_{+a}(g) \equiv\left(\pi_{+}(g), b_{-a}(g)\right),
$$

provides a local trivialisation of the fibre bundle $G \rightarrow F_{+}$. Considering all possible values of the index $a$, we get an atlas of this fibre bundle.

Let $g$ be such an element of $G$ that $\pi_{+}(g) \in U_{+a} \cap U_{+b}$. In this case, using Eq. (2.41), we can write

$$
b_{-a}(g)=b_{-a}\left(s_{+b}(p) b_{-b}(g)\right)=b_{-a}\left(s_{+b}(p)\right) b_{-b}(g),
$$

where $p=\pi_{+}(g)$. Hence we have

$$
b_{-a}(g)=b_{-a b}\left(\pi_{+}(g)\right) b_{-b}(g),
$$

where

$$
b_{-a b} \equiv b_{-a} \circ s_{+b} .
$$

It is clear that the mappings $b_{-a b}, a, b \in A$, are the transition functions of the atlas we have defined. These transition functions are evidently holomorphic.

Let again $\pi_{+}(g) \in U_{+a} \cap U_{+b}$. In this case we have

$$
g=m_{+a}(g) b_{-a}=m_{+b}(g) b_{-b}(g) .
$$

This relation, with the account of Eq. (2.44) gives

$$
m_{+b}(g)=m_{+a}(g) b_{-a b}\left(\pi_{+}(g)\right) .
$$

The groups $\tilde{B}_{ \pm}$have the holomorphic decomposition

$$
\tilde{B}_{ \pm}=\tilde{N}_{ \pm} \tilde{H}
$$

Thus, in our case we can uniquely represent the mapping $b_{-a}$ in the form

$$
b_{-a}=n_{-a} h_{-a},
$$


where $n_{-a}$ and $h_{-a}$ are holomorphic mappings from $\left(\pi_{+}\right)^{-1}\left(U_{+a}\right)$ to the subgroups $\tilde{N}_{-}$and $\tilde{H}$, respectively. Analogously for the transition functions $b_{-a b}$ we have

$$
b_{-a b}=n_{-a b} h_{-a b},
$$

where $n_{-a b}$ and $h_{-a b}$ are holomorphic mappings from $U_{+a} \cap U_{+b}$ to $\tilde{N}_{-}$and $\tilde{H}$, respectively.

From Eq. (2.41) we get the following relations

$$
\begin{gathered}
m_{+a}(g n)=m_{+a}(g), \quad m_{+a}(g h)=m_{+a}(g) \\
n_{-a}(g n)=n_{-a}(g) h_{-a}(g) n\left(h_{-a}(g)\right)^{-1}, \quad n_{-a}(g h)=n_{-a}(g), \\
h_{-a}(g n)=h_{-a}(g), \quad h_{-a}(g h)=h_{-a}(g) h
\end{gathered}
$$

which are valid for any $n \in \tilde{N}_{\text {- }}$ and $\tilde{h} \in H$.

Using Eq. (2.44), for any $g \in G$ such that $p=\pi_{+}(g) \in U_{+a} \cap U_{+b}$, we also obtain

$$
\begin{gathered}
n_{-a}(g)=n_{-a b}(p) h_{-a b}(p) n_{-b}(g)\left(h_{-a b}(p)\right)^{-1}, \\
h_{-a}=h_{-a b}(p) h_{-b}(g) .
\end{gathered}
$$

Proposition 2.4. Any element $g \in\left(\pi_{+}\right)^{-1}\left(U_{+a}\right)=\tilde{N}_{+a} \tilde{B}_{-}$can be uniquely represented in the form

$$
g=m_{+} n_{-} h_{-},
$$

where $m_{+} \in \tilde{N}_{+a}, n_{-} \in \tilde{N}_{-}, h_{-} \in \tilde{H}$. The elements $m_{+}, n_{-}$, and $h_{-}$holomorphically depend on $g$.

Proof. We come to the representation (2.56) putting

$$
m_{+}=m_{+a}(g), \quad n_{-}=n_{-a}(g), \quad h_{-}=h_{-a}(g) .
$$

The uniqueness of the decomposition (2.56) follows directly from the fact that $\tilde{N}_{+} \cap \tilde{N}_{-}=\tilde{N}_{+} \cap \tilde{H}=\tilde{N}_{-} \cap \tilde{H}=\{e\}$.

There takes place the proposition similar to the previous one. 
Proposition 2.5. Any element $g \in\left(\pi_{-}\right)^{-1}\left(U_{-a}\right)=\tilde{N}_{-a} \tilde{B}_{+}$can be uniquely represented in the form

$$
g=m_{-} n_{+} h_{+}
$$

where $m_{-} \in \tilde{N}_{-a}, n_{+} \in \tilde{N}_{+}, h_{+} \in \tilde{H}$. The elements $m_{-}, n_{+}$, and $h_{+}$holomorphically depend on $g$.

We call decompositions (2.56) and (2.58) the modified Gauss decompositions.

2.4. Toda Equations. Now we shall use the modified Gauss decomposition to define the needed $\tilde{H}$-gauge invariant quantities, and to derive the equations they satisfy.

Proposition 2.6. Let $\varphi: M \rightarrow G$ be an arbitrary mapping, and $p \in M$.

(i) There exists an open neighborhood $V_{+}$of the point $p$, such that the mapping $\varphi$ restricted to $V_{+}$has a unique decomposition

$$
\varphi=\mu_{+} \nu_{-} \eta_{-}
$$

where the mapping $\mu_{+}$takes values in $\tilde{N}_{+a}$ for some $a \in A$, while the mappings $\nu_{-}$and $\eta_{-}$take values in $\tilde{N}_{-}$and $\tilde{H}$, respectively.

(ii) There exists an open neighborhood $V_{-}$of the point $p$, such that the mapping $\varphi$ restricted to $V_{-}$has a unique decomposition

$$
\varphi=\mu_{-} \nu_{+} \eta_{+}
$$

where the mapping $\mu_{-}$takes values in $\tilde{N}_{-a}$ for some $a \in A$, while the mappings $\nu_{+}$and $\eta_{+}$take values in $\tilde{N}_{+}$and $\tilde{H}$, respectively.

Proof. The proof is based on the modified Gauss decomposition (2.56). It is clear that we can find $a \in A$ such that $\varphi(p) \in\left(\pi_{+}\right)^{-1}\left(U_{+a}\right)$. Define a mapping $\mu_{+}$by

$$
\mu_{+} \equiv m_{+a} \circ \varphi=s_{+a} \circ \pi_{+} \circ \varphi=s_{+a} \circ \varphi_{+} .
$$

The domain of the mapping $\mu_{+}$is the open set $V_{+} \equiv \varphi_{+}^{-1}\left(U_{+a}\right)$. Introducing now the mappings

$$
\nu_{-} \equiv n_{-a} \circ \varphi, \quad \eta_{-} \equiv h_{-a} \circ \varphi
$$


with the same domain, we come to the required decomposition (2.59).

The second part of the proposition can be proved in the similar way.

Corollary 2.2. For any $p \in M$, there exist an open set $V$, such that $p \in V$, and the mapping $\varphi$ restricted to $V$ possesses simultaneously decompositions (2.59) and (2.60).

Proposition 2.7. If the mapping $\varphi: M \rightarrow G$ satisfies the grading condition, then the mapping $\mu_{-}$is holomorphic and the holomorphic 1 -form ${ }^{\mu_{-} \omega}$ takes values in $\mathfrak{g}_{-1}$; while the mapping $\mu_{+}$is antiholomorphic and the antiholomorphic $1-$ form ${ }^{\mu_{+}} \omega$ takes values in $\mathfrak{g}_{+1}$.

Proof. The required properties of the mappings $\mu_{ \pm}$follow directly from the grading condition.

Let $x_{ \pm}$be some fixed nonzero elements of $\mathfrak{g}_{ \pm 1}$. In the case when we consider the $\mathbb{Z}$-gradation of $\mathfrak{g}$ associated with an integral embedding of $\mathfrak{s l}(2, \mathbb{C})$ into $\mathfrak{g}$, we can take as $x_{ \pm}$the corresponding elements defined by this embedding. Let $\mathcal{O}_{ \pm}$be the orbits of the restriction of the adjoint action of the group $G$ to the subgroup $\tilde{H}$, generated by the elements $x_{ \pm}$. Note that the orbits $\mathcal{O}_{ \pm}$have the following property. If the element $x$ belongs to $\mathcal{O}_{+}\left(\mathcal{O}_{-}\right)$then for any nonzero $c \in \mathbb{C}$ the element $c x$ also belongs to $\mathcal{O}_{+}\left(\mathcal{O}_{-}\right)$. This follows from the fact that the gradation operator $q$ (see Appendix B) generates similarity transformations of the subspaces $\mathfrak{g}_{ \pm 1}$.

Denoting by $\tilde{H}_{ \pm}$the isotropy subgroups of the elements $x_{ \pm}$, we identify the orbits $\mathcal{O}_{ \pm}$with the homogeneous manifolds $\tilde{H} / \tilde{H}_{ \pm}$. More precisely, we establish such identification putting into the correspondence to a coset $h \tilde{H}_{ \pm}$, $h \in \tilde{H}$, the element $x\left(h \tilde{H}_{ \pm}\right) \in \mathcal{O}_{ \pm}$given by

$$
x\left(h \tilde{H}_{ \pm}\right)=\sqrt{-1} \operatorname{Ad}(h) x_{ \pm} .
$$

The factor $\sqrt{-1}$ is introduced in this relation for the future convenience.

Let $\mathcal{O}_{ \pm g}$ be a subset of $T_{g}^{(1,0)}(G)$ defined by

$$
\mathcal{O}_{ \pm g} \equiv L_{g *}\left(\mathcal{O}_{ \pm}\right)
$$

We call a mapping $\varphi: M \rightarrow G$ an admissible mapping, if it satisfies the grading condition, and, moreover, $\varphi_{-*}\left(\partial_{z p}\right) \in \pi_{-*}\left(\mathcal{O}_{-g}\right)$ and $\varphi_{+*}\left(\partial_{\bar{z} p}\right) \in \pi_{+*}\left(\mathcal{O}_{+g}\right)$ for 
any $p \in M$ and $g \in G$ such that $\pi_{ \pm}(g)=p$. Due to the properties of the orbits $\mathcal{O}_{ \pm}$discussed above, this definition does not depend on the choice of the local coordinate $z$. If the mapping $\varphi$ is admissible, then we can write

$$
{ }^{\mu_{-}} \omega=\lambda_{-} d z, \quad{ }_{\mu_{+}} \omega=\lambda_{+} d \bar{z},
$$

where $\mu_{ \pm}$are the mappings arising from the local decompositions (2.59), (2.60), and $\lambda_{ \pm}$are the mappings taking values in $\tilde{H} / \tilde{H}_{ \pm}$. The mappings $\lambda_{ \pm}$are defined in the open set $V$ from Corollary 2.2. It follows from Proposition 2.7, that the mapping $\lambda_{-}$is holomorphic, and the mapping $\lambda_{+}$is antiholomorphic. Let $\gamma_{ \pm}$be local lifts of the mappings $\lambda_{ \pm}$to the group $\tilde{H}$. These mappings are defined in some open set $W \subset V$, and satisfy the relations

$$
\lambda_{ \pm}=\sqrt{-1} \operatorname{Ad}\left(\gamma_{ \pm}\right) x_{ \pm} .
$$

Note that in the case when the groups $\tilde{H}_{ \pm}$are nontrivial, the mappings $\gamma_{ \pm}$ are defined ambiguously, but in any case they can be chosen in such a way that the mapping $\lambda_{-}$would be holomorphic, and the mapping $\lambda_{+}$would be antiholomorphic. In what follows we will use in our consideration such a choice.

Theorem 2.3. Let $\varphi: M \rightarrow G$ be an admissible mapping. There exists a local $\tilde{H}$-gauge transformation that transforms a connection ${ }^{\varphi} \omega$ to the connection ${ }^{\varphi^{\prime}} \omega$ of the form

$$
{ }^{\prime} \omega=\left(\sqrt{-1} x_{-}+\left({ }^{\gamma} \omega\right)_{-}\right) d z+\sqrt{-1} \operatorname{Ad}\left(\gamma^{-1}\right) x_{+} d \bar{z},
$$

where $\gamma$ is the mapping taking values in $\tilde{H}$ and given by

$$
\gamma \equiv \gamma_{+}^{-1} \eta \gamma_{-}
$$

with

$$
\eta \equiv \eta_{-} \eta_{+}^{-1}
$$

Proof. Using representation (2.60) and Proposition 2.1, we can write

$$
\varphi_{\omega}={ }^{\mu_{-} \nu_{+} \eta_{+}} \omega=\operatorname{Ad}\left(\eta_{+}^{-1} \nu_{+}^{-1}\right)\left({ }^{\mu_{-}} \omega\right)+\operatorname{Ad}\left(\eta_{+}^{-1}\right)\left({ }^{\nu+} \omega\right)+{ }^{\eta_{+}} \omega .
$$


In the same way, representation (2.59) gives

$$
{ }^{\varphi} \omega={ }^{\mu_{+} \nu_{-} \eta_{-}} \omega=\operatorname{Ad}\left(\eta_{-}^{-1} \nu_{-}^{-1}\right)\left({ }^{\mu_{+}} \omega\right)+\operatorname{Ad}\left(\eta_{-}^{-1}\right)\left({ }^{\nu_{-}} \omega\right)+{ }^{\eta_{-}} \omega
$$

and since the form ${ }^{\mu_{-}} \omega$ is holomorphic, and the form ${ }^{\mu_{+}} \omega$ is antiholomorphic,

$$
\left({ }^{\mu_{-}} \omega\right)_{+}=0, \quad\left({ }^{\mu_{+}} \omega\right)_{-}=0 .
$$

Taking into account these relations, we come to the following consequences of Eqs. (2.70) and (2.71):

$$
\left({ }^{\varphi} \omega\right)_{+}=\operatorname{Ad}\left(\eta_{+}^{-1}\right)\left({ }^{\nu_{+}} \omega\right)_{+}+\left({ }^{\eta_{+}} \omega\right)_{+}, \quad\left({ }^{\varphi} \omega\right)_{-}=\operatorname{Ad}\left(\eta_{-}^{-1}\right)\left({ }^{\nu_{-}} \omega\right)_{-}+\left({ }^{\eta_{-}} \omega\right)_{-} .
$$

Consider now the mapping

$$
\kappa \equiv \mu_{+}^{-1} \mu_{-} .
$$

Proposition 2.1 and Corollary 2.1 provide the relation

$$
{ }^{\kappa} \omega={ }^{\mu_{-}} \omega-\operatorname{Ad}\left(\kappa^{-1}\right)\left({ }^{\mu_{+}} \omega\right) .
$$

Using Eq. (2.65), we come from this relation to the equalities

$$
\left({ }^{\kappa} \omega\right)_{+}=-\operatorname{Ad}\left(\kappa^{-1}\right) \lambda_{+}, \quad\left({ }^{\kappa} \omega\right)_{-}=\lambda_{-} .
$$

Thanks to decompositions (2.59) and (2.60), we conclude that the mapping $\kappa$ can be also represented as

$$
\kappa=\nu_{-} \eta \nu_{+}^{-1}
$$

Representation (2.77) leads to the equality

$$
{ }^{\kappa} \omega=\operatorname{Ad}\left(\nu_{+} \eta^{-1}\right)\left({ }^{\nu_{-}} \omega-\eta^{-1} \omega-\operatorname{Ad}(\eta)\left({ }^{\nu_{+}} \omega\right)\right),
$$

which results in the formula

$$
\operatorname{Ad}\left(\eta \nu_{+}^{-1}\right) \lambda_{-}=\left(\nu_{-} \omega\right)_{-}-\left(\eta^{-1} \omega\right)_{-}-\operatorname{Ad}(\eta)\left({ }^{\nu_{+}} \omega\right)_{-} .
$$

Taking the $\mathfrak{n}_{-}-$component of Eq. (2.79), we come to the relation

$$
\left(\nu_{-} \omega\right)_{-}=\operatorname{Ad}(\eta) \lambda_{-} .
$$

Similarly, it follows from Eq. (2.78) that

$$
-\operatorname{Ad}\left(\eta^{-1} \nu_{-}^{-1}\right) \lambda_{+}=\operatorname{Ad}\left(\eta^{-1}\right)\left({ }^{\nu} \omega\right)_{+}+\left({ }^{\eta} \omega\right)_{+}-\left({ }^{\nu_{+}} \omega\right)_{+},
$$


and $\mathfrak{n}_{+}-$component of this relation is

$$
\left({ }^{\nu_{+}} \omega\right)_{-}=\operatorname{Ad}\left(\eta^{-1}\right) \lambda_{+}
$$

Substitution of Eqs. (2.80) and (2.82) into Eq. (2.73) gives

$(2.83)\left({ }^{\varphi} \omega\right)_{+}=\operatorname{Ad}\left(\eta_{-}^{-1}\right) \lambda_{+}+\left(\eta_{+} \omega\right)_{+}$

$$
\left({ }^{\varphi} \omega\right)_{-}=\operatorname{Ad}\left(\eta_{+}^{-1}\right) \lambda_{-}+\left({ }^{\eta_{-}} \omega\right)_{-} .
$$

Taking into account Eq. (2.66), we come to the relations

$$
\begin{aligned}
& \left({ }^{\varphi} \omega\right)_{+}=\sqrt{-1} \operatorname{Ad}\left(\eta_{-}^{-1} \gamma_{+}\right) x_{+}+\left({ }^{\eta_{+}} \omega\right)_{+}, \\
& \left({ }^{\varphi} \omega\right)_{-}=\sqrt{-1} \operatorname{Ad}\left(\eta_{+}^{-1} \gamma_{-}\right) x_{-}+\left({ }^{\eta_{-}} \omega\right)_{-} .
\end{aligned}
$$

Performing now the gauge transformation defined by the mapping $\eta_{+}^{-1} \gamma_{-}$, we arrive at representation (2.67) with the mapping $\gamma$ given by Eq. (2.68).

For the case of a linear group one can rewrite relation (2.67) in the form

$$
\varphi_{\omega}=\left(\sqrt{-1} x_{-}+\gamma^{-1} \partial_{z} \gamma\right) d z+\sqrt{-1}\left(\gamma^{-1} x_{+} \gamma\right) d \bar{z},
$$

which allows to present the zero curvature condition (2.3) in the form

$$
\partial_{\bar{z}}\left(\gamma^{-1} \partial_{z} \gamma\right)=\left[\gamma^{-1} x_{+} \gamma, x_{-}\right] .
$$

In a general case, instead of Eq. (2.86) we have the following equation

$$
\partial_{\bar{z}}\left(\left({ }^{\gamma} \omega\right)_{-}\right)=\left[\operatorname{Ad}\left(\gamma^{-1}\right) x_{+}, x_{-}\right],
$$

which is given here without proof. In concrete examples we usually deal with linear groups and use Eq. (2.86).

The system of equations for parameters of the group $\tilde{H}$, which follows from Eq. (2.86), is called the Toda equations. In the case of the canonical gradation, the subgroup $\tilde{H}$ coincide with some Cartan subgroup $H$ of $G$, and is, by this reason, an abelian subgroup. The corresponding equations in this case are called the abelian Toda equations. In the case of a general $\mathbb{Z}$-gradation associated with an integral embedding of $\mathfrak{s l}(2, \mathbb{C})$ into $\mathfrak{g}$, the subgroup $\tilde{H}$ is not necessarily abelian, and we deal with the abelian Toda equations and their nonabelian versions.

Let us derive the concrete form of the abelian Toda equations. These equations will be used in section 4 to prove the generalised Plücker relations. In 
the case under consideration, we can locally parametrise the mapping $\gamma$ by the set of complex functions $f_{i}$ as

$$
\gamma=\exp \left(\sum_{i=1}^{r} f_{i} h_{i}\right)
$$

where $r=\operatorname{rank} \mathfrak{g}$, and the elements $h_{i} \in \mathfrak{h}$ correspond to the simple roots of $\mathfrak{g}$ (see appendix B). Choose as the elements $x_{ \pm} \in \mathfrak{g}_{ \pm 1}$ the elements describing the corresponding principal embedding of $\mathfrak{s l}(2, \mathbb{C})$ in $\mathfrak{g}$. The concrete form of such elements is given by Eq. (B.42). Using Eq. (B.11), we get the relation

$$
\gamma^{-1} x_{+} \gamma=\sum_{i=1}^{r}\left(2 k_{i}\right)^{1 / 2} \exp \left[-(k f)_{i}\right] x_{+i}
$$

where

$$
(k f)_{i} \equiv \sum_{j=1}^{r} k_{i j} f_{j}
$$

From this relation we immediately have

$$
\left[\gamma^{-1} x_{+} \gamma, x_{-}\right]=\sum_{i=1}^{r} 2 k_{i} \exp \left[-(k f)_{i}\right] h_{i} .
$$

From the other hand, it is clear that

$$
\partial_{\bar{z}}\left(\gamma^{-1} \partial_{z} \gamma\right)=\sum_{i=1}^{r}\left(\partial_{\bar{z}} \partial_{z} f_{i}\right) h_{i}
$$

Thus, in the case under consideration, equations (2.86) can be reduced to the system

$$
\partial_{\bar{z}} \partial_{z} f_{i}=2 k_{i} \exp \left[-(k f)_{i}\right], \quad i=1, \ldots, r .
$$

Introducing the functions

$$
u_{i} \equiv(k f)_{i}-\ln \left(2 k_{i}\right),
$$

we rewrite equations (2.93) in the form

$$
\partial_{\bar{z}} \partial_{z} u_{i}=\sum_{j=1}^{r} k_{i j} \exp \left(-u_{j}\right),
$$

which is standard for the abelian Toda equations. 
2.5. Gauge Invariance and Dependence on Lifts. Consider now the behaviour of the mapping $\gamma$ under the $\tilde{H}$-gauge transformation. Let $\varphi^{\prime}=\varphi \psi$, where the mapping $\psi$ takes values in $\tilde{H}$. It is clear that to define the mapping $\gamma^{\prime}$ corresponding to the mapping $\varphi^{\prime}$, we can use the same modified Gauss decompositions, which we have used for the construction of the mapping $\gamma$. From the relations (2.61) and (2.51) we have $\mu_{+}^{\prime}=\mu_{+}$. Using the same lift from $\tilde{H} / \tilde{H}_{+}$to $\tilde{H}$, which was used in transition from the mapping $\mu_{+}$to the mapping $\gamma_{+}$, we obtain $\gamma_{+}^{\prime}=\gamma_{+}$. From the other hand, relations (2.62) and (2.53) give $\eta_{-}^{\prime}=\eta_{-} \psi$. In the similar way we have $\gamma_{-}^{\prime}=\gamma_{-}$, and $\eta_{+}^{\prime}=\eta_{+} \psi$. Thus, it follows from Eq. (2.68) that $\gamma^{\prime}=\gamma$. Just in this sense the mapping $\gamma$ is $\tilde{H}$-gauge invariant.

The last question we are going to consider in this section, is the dependence of the mapping $\gamma$ on the choice of modified Gauss decompositions and local lifts from $\tilde{H} / \tilde{H}_{ \pm}$to $\tilde{H}$. Let we have two local decompositions of the mapping $\varphi:$

$$
\varphi=\mu_{+} \nu_{-} \eta_{-}, \quad \varphi=\mu_{+}^{\prime} \nu_{-}^{\prime} \eta_{-}^{\prime}
$$

which are obtained with the help of the modified Gauss decompositions corresponding to the indexes $a$ and $b$, respectively. Using Eqs. (2.47) and (2.50), we get

$$
\mu_{+}^{\prime}=m_{+b} \circ \varphi=\mu_{+} \nu_{-a b} \eta_{-a b}
$$

where

$$
\nu_{-a b} \equiv n_{-a b} \circ \varphi_{+}, \quad \eta_{-a b} \equiv h_{-a b} \circ \varphi_{+}
$$

It is evident that the mappings $\nu_{-a b}$ and $\eta_{-a b}$ are holomorphic, and take values in $\tilde{N}_{-}$and $\tilde{H}$, respectively. From Eq. (2.97) it follows that

$$
\mu_{+\omega}^{\prime}=\operatorname{Ad}\left(\eta_{-a b}^{-1}\right)\left(\operatorname{Ad}\left(\nu_{-a b}^{-1}\right)\left({ }^{\mu_{+}} \omega\right)+{ }^{\nu_{-a b}} \omega\right)+{ }^{\eta_{-a b}} \omega
$$

Since the forms ${ }^{\mu_{+}} \omega$ and ${ }^{\mu_{+}^{\prime}} \omega$ take values in $\mathfrak{g}_{+1}^{\prime}$, we actually have the relation

$$
\mu_{+\omega}^{\prime}=\operatorname{Ad}\left(\eta_{-a b}^{-1}\right)\left({ }^{\mu_{+}} \omega\right)
$$


Taking into account Eqs. (2.65) and (2.66), we get

$$
\operatorname{Ad}\left(\gamma_{+}^{\prime}\right) x_{+}=\operatorname{Ad}\left(\eta_{-a b} \gamma_{+}\right) x_{+}
$$

It follows from this relation that

$$
\gamma_{+}^{\prime}=\eta_{-a b}^{-1} \gamma_{+} \xi_{+}
$$

where the mapping $\xi_{+}$takes values in $\tilde{H}_{+}$. It is clear that the mapping $\xi_{+}$is antiholomorphic.

Further, Eq. (2.55) allows to write

$$
\eta_{-}=\eta_{-a b} \eta_{-}^{\prime}
$$

Combining now Eqs. (2.105) and (2.103), we obtain

$$
\gamma_{+}^{\prime-1} \eta_{-}^{\prime}=\xi_{+}^{-1} \gamma_{+}^{-1} \eta_{-}
$$

In the similar way we have

$$
\eta_{+}^{\prime-1} \gamma_{-}^{\prime}=\eta_{+}^{-1} \gamma_{-} \xi_{-}
$$

where $\xi_{-}$is a holomorphic mapping taking values in $\tilde{H}_{-}$. Finally, we come to the relation

$$
\gamma^{\prime}=\xi_{+}^{-1} \gamma \xi_{-}
$$

Resuming our consideration, we can say that any admissible mapping $\varphi$ leads to a set $\left\{\gamma_{i}\right\}_{i \in I}$ of local solutions of equations (2.86). These solutions, in the overlaps of their domains, are connected by the relations

$$
\gamma_{i}=\xi_{+i j}^{-1} \gamma_{j} \xi_{-i j}
$$

where the mappings $\xi_{-i j}$ are holomorphic and take values in $\tilde{H}_{-}$, while the mappings $\xi_{+i j}$ are antiholomorphic and take values in $\tilde{H}_{+}$. Note here that Eq. (2.105) describes symmetry transformations of equations (2.86). For the case of the canonical gradation, the subgroups $\tilde{H}_{ \pm}$are discrete and isomorphic to $\underbrace{\mathbb{Z}_{2} \times \cdots \times \mathbb{Z}_{2}}_{r}$. 


\section{Construction of Solutions and Reality Condition}

In the previous section we have shown that any admissible mapping $\varphi$ : $M \rightarrow G$ allows to construct a set of local solutions of equations (2.86). From the first glance, the problem of constructing admissible mappings is rather complicated. In fact, any admissible mapping $\varphi$ is defined by the corresponding mappings $\varphi_{+}$and $\varphi_{-}$up to an $\tilde{H}$-gauge transformation. In other words the following proposition is valid.

Proposition 3.1. Let $\varphi$ and $\varphi^{\prime}$ be two mappings from $M$ to $G$. The equalities

$$
\varphi_{+}^{\prime}=\varphi_{+}, \quad \varphi_{-}^{\prime}=\varphi_{-}
$$

are valid if and only if $\varphi^{\prime}=\varphi \psi$, where the mapping $\psi$ takes values in $\tilde{H}$.

Proof. The assertion of the propositions follows directly from the fact that $\tilde{B}_{-} \cup \tilde{B}_{+}=\tilde{H}$.

Since the mapping $\gamma$ is $\tilde{H}$-gauge invariant, we conclude that this mapping can be constructed using only information contained in the mappings $\varphi_{ \pm}$. In practice, it can be done in the following way.

Let $\sigma$ be an involutive antilinear automorphism of $\mathfrak{g}$. It is known that the mapping $\sigma$ defines a real form $\mathfrak{g}_{\sigma}$ of the Lie algebra $\mathfrak{g}$ by

$$
\mathfrak{g}_{\sigma} \equiv\{x \in \mathfrak{g} \mid \sigma(x)=x\} .
$$

Introduce the $\sigma$-hermitian conjugation as a mapping from $\mathfrak{g}$ to $\mathfrak{g}$ which assigns to any element $x \in \mathfrak{g}$ the element $x^{\star} \in \mathfrak{g}$, defined by

$$
x^{\star} \equiv-\sigma(x)
$$

It is clear that the $\sigma$-hermitian conjugation is an antilinear antiautomorphism of $\mathfrak{g}$ satisfying the condition

$$
\left(x^{\star}\right)^{\star}=x
$$

for any $x \in \mathfrak{g}$. An element $x \in \mathfrak{g}$ is said to be $\sigma$-hermitian if $x^{\star}=x$, and it is said to be $\sigma$-antihermitian if $x^{\star}=-x$. The subalgebra $\mathfrak{g}_{\sigma}$ is formed by all $\sigma$-antihermitian elements of $\mathfrak{g}$. 
Suppose that $\sigma$ can be extended to an antiholomorphic automorphism $\Sigma$ of the group $G$. In this case we have $\Sigma^{2}=\operatorname{id}_{G}$. The $\Sigma$-hermitian conjugation in $G$ is defined then as a mapping $g \in G \rightarrow g^{\star} \in G$, where

$$
g^{\star} \equiv \Sigma\left(g^{-1}\right)=(\Sigma(g))^{-1} .
$$

It is evident that $\Sigma$-hermitian conjugation is an antiholomorphic antiautomorphism of $G$ satisfying the condition

$$
\left(g^{\star}\right)^{\star}=g
$$

for any $g \in G$. An element $g \in G$ is called $\Sigma$-hermitian if $g^{\star}=g$, and it is called $\Sigma$-unitary if $g^{\star}=g^{-1}$. In the case when the mapping $\Sigma$ is originated from the mapping $\sigma$, giving a compact real form of $\mathfrak{g}$, we say simply 'hermitean' and 'unitary'. The real Lie group $G_{\sigma}$ corresponding to the real form $\mathfrak{g}_{\sigma}$, is formed by all $\Sigma$-unitary elements of $G$.

Suppose also that the mapping $\sigma$ has the property

$$
\sigma\left(\mathfrak{g}_{m}\right)=\mathfrak{g}_{-m}, \quad m \in \mathbb{Z} .
$$

In this case one has

$$
\tilde{H}^{\star}=\tilde{H}, \quad\left(\tilde{N}_{ \pm}\right)^{\star}=\tilde{N}_{\mp} .
$$

A representation $\rho: G \rightarrow G L(V)$ of the group $G$ in the linear space $V$ over the field $\mathbb{C}$ is called $\Sigma$-unitary, if the space $V$ is equipped with a hermitean scalar product $\langle$,$\rangle such that$

$$
\rho(g)^{\star}=\rho\left(g^{\star}\right),
$$

where $\star$ in the left hand side means the hermitean conjugation with respect to the scalar product $\langle$,$\rangle . It can be shown that the representation \rho$ is $\Sigma$-hermitean if and only if the restriction of $\rho$ to the real Lie group $G_{\sigma}$ is unitary.

Let us consider now an arbitrary $\Sigma$-unitary representation of the group $G$ in a linear space $V$. Denote by $V_{+}$the subspace of $V$ formed by all elements $v \in V$, such that

$$
\rho(g) v=v
$$


for all $g \in \tilde{N}_{+}$. For any mapping $\varphi: M \rightarrow G$ and any vectors $u, v \in V$ we denote by $\langle u, \varphi v\rangle$ the mapping from $M$ to $\mathbb{C}$, defined as

$$
\langle u, \varphi v\rangle(p) \equiv\langle u, \rho(\varphi(p)) v\rangle \text {. }
$$

Theorem 3.1. For any $u, v \in V_{+}$the following relation is valid:

$$
\langle u, \gamma v\rangle=\left\langle u,\left(\mu_{+} \gamma_{+}\right)^{-1}\left(\mu_{-} \gamma_{-}\right) v\right\rangle
$$

Proof. Comparing Eqs. (2.74) and (2.77), we get

$$
\nu_{-} \eta \nu_{+}^{-1}=\mu_{+}^{-1} \mu_{-} .
$$

Now, using the definition (2.68) of the mapping $\gamma$, we come to the relation

$$
\nu_{-}^{\prime} \gamma \nu_{+}^{\prime-1}=\left(\mu_{+} \gamma_{+}\right)^{-1}\left(\mu_{-} \gamma_{-}\right)
$$

where

$$
\nu_{-}^{\prime} \equiv \gamma_{+}^{-1} \nu_{-} \gamma_{+}, \quad \nu_{+}^{\prime} \equiv \gamma_{-}^{-1} \nu_{+} \gamma_{-} .
$$

Then, the validity of Eq. (3.12) follows from Eq. (3.14) and the definition of $V_{+}$.

Thus, we can find some matrix elements of the linear operators corresponding to the mapping $\gamma$ in a $\Sigma$-unitary representation of the group $G$, using only the mappings $\mu_{ \pm}$, which in their turn are determined only by the mappings $\varphi_{ \pm}$. It is natural to suppose here that using a reach enough set of representations, one will be able to recover the mapping $\gamma$ from the mappings $\varphi_{ \pm}$. It is likely that for the case of simple Lie groups, it is enough to use only fundamental representations, that is confirmed by the consideration of concrete examples.

Let us consider now a special class of the solutions which will be called real solutions. To this end, introduce two antiholomorphic mappings $\Sigma_{ \pm}: F_{ \pm} \rightarrow$ $F_{\mp}$ defined by

$$
\Sigma_{ \pm}\left(g \tilde{B}_{\mp}\right) \equiv \Sigma(g) \tilde{B}_{ \pm}
$$

It is easy to get convinced that the mappings $\Sigma_{ \pm}$are defined correctly. Directly from the definition of these mappings, we get the equalities

$$
\Sigma_{+} \circ \pi_{+}=\pi_{-} \circ \Sigma, \quad \Sigma_{-} \circ \pi_{-}=\pi_{+} \circ \Sigma \text {. }
$$


Moreover, these mappings are mutually inverse:

$$
\Sigma_{+} \circ \Sigma_{-}=\mathrm{id}_{F_{-}}, \quad \Sigma_{-} \circ \Sigma_{+}=\mathrm{id}_{F_{+}} .
$$

A mapping $\varphi: M \rightarrow G$ is said to satisfy the reality condition if

$$
\Sigma_{+} \circ \varphi_{+}=\varphi_{-}
$$

that can be also written as

$$
\Sigma_{-} \circ \varphi_{-}=\varphi_{+}
$$

Proposition 3.2. A mapping $\varphi: M \rightarrow G$ satisfies the reality condition if and only if

$$
\Sigma \circ \varphi=\varphi \psi
$$

where the mapping $\psi$ takes values in $\tilde{H}$.

Proof. Let $\varphi$ satisfies the reality condition. Using Eq. (3.17) in Eq. (3.19), we get

$$
\pi_{-} \circ \Sigma \circ \varphi=\pi_{-} \circ \varphi
$$

Hence, $\Sigma \circ \varphi=\varphi \psi$, where $\psi$ takes values in $\tilde{B}_{+}$. In the similar way, from Eq. (3.20) we see that $\psi$ takes values in $\tilde{B}_{-}$. Since $\tilde{B}_{+} \cap \tilde{B}_{-}=\tilde{H}$, we conclude that $\psi$ takes values in $\tilde{H}$.

The inverse statement of the proposition is evident.

A mapping $\psi: M \rightarrow G$ is called $\Sigma$-hermitean if for any $p \in M$ the mapping $\psi(p)$ is a $\Sigma$-hermitean element of $G$.

Proposition 3.3. The mapping $\psi$ from proposition 3.2 is $\Sigma$-hermitean.

Proof. Since $\Sigma^{2}=\mathrm{id}_{G}$, from Eq. (3.21) we get

$$
\varphi=(\Sigma \circ \varphi)(\Sigma \circ \psi)
$$

that can be written as

$$
\Sigma \circ \varphi=\varphi(\Sigma \circ \psi)^{-1}
$$


Comparing Eq. (3.24) with Eq. (3.21) we have

$$
(\Sigma \circ \psi)^{-1}=\psi
$$

Hence, the mapping $\psi$ is $\Sigma$-hermitean.

Proposition 3.4. The reality condition is $\tilde{H}$-gauge invariant.

Proof. Let a mapping $\varphi$ satisfies the reality condition, and $\varphi^{\prime}=\varphi \xi$ with $\xi$ taking values in $\tilde{H}$, be a gauge transformed mapping. Using Eq. (3.21) we have

$$
\Sigma \circ \varphi^{\prime}=\varphi^{\prime} \circ \psi^{\prime}
$$

where

$$
\psi^{\prime}=\xi^{-1} \psi(\Sigma \circ \xi)
$$

Since $\Sigma(\tilde{H})=\tilde{H}$, the mapping $\psi^{\prime}$ satisfies the reality condition.

Let $s_{+a}, a \in A$, be a family of local holomorphic sections of the fibre bundle $G \rightarrow F_{+}$with the properties described in proposition 4.3. The mapping $\Sigma$ allows us to construct the corresponding family of local holomorphic sections of the fibre bundle $G \rightarrow F_{-}$. For each open set $U_{+a}$ we define the open set $U_{-a}$ by

$$
U_{-a} \equiv \Sigma_{+}\left(U_{+a}\right)
$$

Using Eqs. (3.17) and (3.18), it is easy to get convinced that for any $a \in A$, the mapping $s_{-a}: U_{-a} \rightarrow G$ given by

$$
s_{-a} \equiv \Sigma \circ s_{+a} \circ \Sigma_{-}
$$

is a local holomorphic section of the fibre bundle $G \rightarrow F_{-}$. Since $\Sigma\left(\tilde{N}_{+}\right)=\tilde{N}_{-}$, we also have

$$
\Sigma_{*} \mathcal{N}_{+}=\mathcal{N}_{-}
$$

hence, the set $s_{-a}\left(U_{-a}\right)$ is an integral manifold of the distribution $\mathcal{N}_{-}$. Thus, we get a family of holomorphic sections of the fibre bundle $G \rightarrow F_{-}$with the required properties. Now, to construct the mappings needed to define 
the mapping $\gamma$, we will use for any section $s_{+a}$ the corresponding section $s_{-a}$ defined with the help of the procedure described above.

Proposition 3.5. If a mapping $\varphi$ satisfies the reality condition, then the mappings $\mu_{+}$and $\mu_{-}$entering proposition 2.6 are connected by

$$
\mu_{\mp}=\Sigma \circ \mu_{ \pm} .
$$

Proof. Recall that the mappings $\mu_{ \pm}$are given by

$$
\mu_{ \pm}=s_{ \pm a} \circ \varphi_{ \pm}
$$

From this relation we have

$$
\mu_{\mp}=\Sigma \circ s_{ \pm a} \circ \Sigma_{\mp} \circ \varphi_{\mp}=\Sigma \circ s_{ \pm a} \circ \varphi_{ \pm}=\Sigma \circ \mu_{ \pm} .
$$

This chain of equalities provides the assertion of the proposition.

Proposition 3.6. If a mapping $\varphi$ satisfies the reality condition, then the mappings $\gamma_{ \pm}$satisfying Eq. (2.66) can be chosen in such a way that

$$
\Sigma \circ \gamma_{ \pm}=\gamma_{\mp} .
$$

Proof. First, let us show that the mappings $\lambda_{+}$and $\lambda_{-}$are connected by

$$
\lambda_{-}=\sigma \circ \lambda_{+}
$$

From Eq. (3.31) we have

$$
{ }^{\mu_{-}} \omega=\left(\mu_{+}^{*} \circ \Sigma^{*}\right) \theta .
$$

Now, using the equality (A.33), we get

$$
{ }^{\mu_{-}} \omega(x)=\Sigma^{*} \theta\left(\mu_{+*}(x)\right)=\sigma\left({ }^{\mu_{+}} \omega(\bar{x})\right)
$$

for any $x \in T_{p}^{\mathbb{C}}(M), p \in M$. In particular, we can write the equality

$$
{ }^{\mu_{-}} \omega\left(\partial_{z}\right)=\sigma\left({ }^{\mu_{+}} \omega\left(\partial_{\bar{z}}\right)\right),
$$

which directly leads to Eq. (3.35).

From Eqs. (3.35) it follows that

$$
\operatorname{Ad}\left(\gamma_{-}\right) x_{-}=\left(\sigma \circ \operatorname{Ad}\left(\gamma_{+}\right)\right) x_{+} .
$$


Suppose now that the elements $x_{ \pm}$entering Eq. (2.66) are chosen in such a way that

$$
\sigma\left(x_{+}\right)=-x_{-}
$$

Taking into account relation (A.10), we conclude that one can choose the mappings $\gamma_{+}$and $\gamma_{-}$satisfying Eq. (3.34).

Theorem 3.2. If a mapping $\varphi$ satisfies the reality condition, then the mapping $\gamma$ can be chosen to be $\Sigma$-hermitean.

Proof. From proposition 3.2, using Eqs. (2.59) and (2.60), we get

$$
\Sigma \circ \varphi=\left(\Sigma \circ \mu_{+}\right)\left(\Sigma \circ \nu_{-}\right)\left(\Sigma \circ \eta_{-}\right)=\mu_{-} \nu_{+} \eta_{+} \psi \text {. }
$$

Since decomposition (2.60) is unique, we have

$$
\Sigma \circ \eta_{-}=\eta_{+} \psi
$$

and hence, can write the equality

$$
\eta_{-}=\left(\eta_{+}^{-1}\right)^{\star} \psi^{-1}
$$

which leads to the relation

$$
\eta=\left(\eta_{+}^{-1}\right)^{\star} \psi^{-1} \eta_{+}^{-1}
$$

As it follows from proposition 3.3, the mapping $\psi$ is $\Sigma$-hermitean, thus the mapping $\eta$ is also $\Sigma$-hermitean. Taking into account proposition 3.6 and the definition of the mapping $\gamma$, we conclude that it can be chosen to be $\Sigma$-hermitean.

Thus, we can say that using the mappings $\varphi$ satisfying the reality condition, one can construct hermitean, in a sense real, solutions of the equations (2.86). Since the solutions are actually determined by the mappings $\varphi_{ \pm}$, then to get real solutions of the equations under consideration, we should choose the mappings $\varphi_{ \pm}$satisfying relation (3.19). Note that, in general, not any pair of the mappings $\varphi_{ \pm}$can be used for the construction of a solution. Actually, there are mappings $\varphi_{ \pm}$which do not correspond to any mapping $\varphi$. We call mappings $\varphi_{ \pm}: M \rightarrow F_{ \pm}$consistent if there is a mapping $\varphi: M \rightarrow G$ such that $\varphi_{ \pm}=\pi_{ \pm} \circ \varphi$. 
Suppose now that the involutive antilinear automorphism $\sigma$ is defined by Eq. (B.9), so that the corresponding real form $\mathfrak{u}$ of $\mathfrak{g}$ is compact. In this case the gradation operator is $\sigma$-hermitean, and Eq. (3.7) is valid.

Theorem 3.3. If mappings $\varphi_{ \pm}: M \rightarrow F_{ \pm}$satisfy relation (3.19), then they are consistent.

Proof. It is enough to show that if two points $p_{+} \in F_{+}$and $p_{-} \in F_{-}$are connected by the relation

$$
p_{-}=\Sigma_{+}\left(p_{+}\right)
$$

then there exists an element $g \in G$ such that

$$
p_{+}=\pi_{+}(g), \quad p_{-}=\pi_{-}(g)
$$

This fact can be proved using the Iwasawa decomposition (B.18). Let $g^{\prime}$ be any element of $G$, such that $\pi_{+}\left(g^{\prime}\right)=p_{+}$. This element can be written as

$$
g^{\prime}=u n_{+} a
$$

where $u \in U, n_{+} \in N_{+}$and $a \in A^{*}$, and the subgroups $U, N_{+}$and $A^{*}$ are defined in appendix B. We have $N_{+} A \subset B_{+} \subset \tilde{B}_{+}$, hence $\pi_{+}(u)=p_{+}$. Using now Eq. (3.17) one gets

$$
\left(\Sigma_{+} \circ \pi_{+}\right)(u)=\left(\pi_{-} \circ \Sigma\right)(u)=\pi_{-}(u)=\Sigma_{+}\left(p_{+}\right)
$$

Thus, the element $u$ can be taken as the element $g$ we are looking for.

The generalisation of the results proved in this section and in the previous one, to the case of the semi-integral embeddings of $\mathfrak{s l}(2, \mathbb{C})$ into $\mathfrak{g}$ is straightforward, and can be performed following the consideration given in Ref. [18]. Recall that in that case we deal with $\mathbb{Z} / 2$-gradations of $\mathfrak{g}$ (see appendix $B$ ).

\section{Toda Fields and Generalised Plücker Relations}

In this section we give a derivation of the generalised Plücker relations. Recall first some definitions. Let $M$ be a complex manifold, and $J^{M}$ be the 
corresponding complex structure on $M$. A metric $g$ on $M$ is called hermitean if

$$
g\left(J^{M} X, J^{M} Y\right)=g(X, Y)
$$

for all vector fields $X, Y$ on $M$. Let $g$ be a hermitean metric on $M$. The 2-form $\Phi$ defined by

$$
\Phi(X, Y) \equiv g\left(X, J^{M} Y\right)
$$

for all vector fields $X, Y$ on $M$ is called the fundamental form associated with $g$. A hermitean metric $g$ on $M$ is called a Kähler metric if the fundamental form $\Phi$ associated with $g$ is closed, i.e.,

$$
d \Phi=0 .
$$

The fundamental form $\Phi$ associated with a hermitean metric $g$ can be locally represented as

$$
\Phi=-2 \sqrt{-1} \partial \bar{\partial} K,
$$

where $K$ is a real-valued function. The function $K$ entering Eq. (4.4), is called a Kähler potential of the hermitean metric $g$.

Let $z^{i}$ be local coordinates in $M$; introduce the notation

$$
\partial_{z^{i}} \equiv \partial / \partial z^{i}, \quad \partial_{\bar{z}^{i}} \equiv \partial / \partial \bar{z}^{i} .
$$

For any hermitean metric $g$ on $M$ we have

$$
g\left(\partial_{z^{i}}, \partial_{\bar{z}^{j}}\right)=\overline{g\left(\partial_{z^{j}}, \partial_{\bar{z}^{i}}\right)}
$$

while

$$
g\left(\partial_{z^{i}}, \partial_{z^{j}}\right)=0, \quad g\left(\partial_{\bar{z}^{i}}, \partial_{\bar{z}^{j}}\right)=0 .
$$

Hence, we can write

$$
g=\sum_{i, j} g_{i \bar{j}}\left(d z^{i} \otimes d \bar{z}^{j}+d \bar{z}^{j} \otimes d z^{i}\right) \equiv 2 \sum_{i, j} g_{i \bar{j}} d z^{i} d \bar{z}^{j},
$$

where

$$
g_{i \bar{j}} \equiv g\left(\partial_{z^{i}}, \partial_{\bar{z}^{j}}\right)
$$


For the fundamental form $\Phi$ associated with $g$, we get the representation

$$
\Phi=-2 \sqrt{-1} \sum_{i, j} g_{i \bar{j}} d z^{i} \wedge d \bar{z}^{j} .
$$

Note also that the expression for the Ricci curvature tensor $R$ in the case under consideration has the form [17]

$$
R=2 \sqrt{-1} \partial \bar{\partial} \ln \mathcal{G}
$$

where $\mathcal{G}$ is the determinant of the matrix $\left(g_{i \bar{j}}\right)$.

Let $V$ be a complex linear space, and $\mathbb{P}(V)$ be the projective space associated with $V$. In other words, $\mathbb{P}(V)$ is the set of all lines (one-dimensional subspaces) in $V$. Denote by pr the canonical projection from the set $V^{\prime} \equiv V-\{0\}$ onto $\mathbb{P}(V)$. Suppose that $V$ is endowed with a hermitean scalar product $\langle$,$\rangle . Let$ $F$ be the function on $V^{\prime}$ given by

$$
F(v) \equiv \ln \|v\|^{2},
$$

where $\|v\|^{2} \equiv\langle v, v\rangle$. The Fubini-Study metric on $\mathbb{P}(V)$ is defined by its associated fundamental form $\Phi^{F S}$, which is a unique form satisfying the relation

$$
\operatorname{pr}^{*} \Phi^{F S}=-2 \sqrt{-1} \partial \bar{\partial} F .
$$

Let $G$ be a complex semisimple Lie group of rank $r$, $\mathfrak{g}$ be its Lie algebra endowed with the canonical $\mathbb{Z}$-gradation, and $\mathfrak{h}$ be the corresponding Cartan subalgebra. Consider the Borel subgroup $B_{+}$, corresponding to the Borel subalgebra $\mathfrak{b}_{+}$defined by Eq. (B.14). Let $\varphi_{-}$be a holomorphic mapping from complex manifold $M$ of complex dimension one to the flag manifold $F_{-}=G / B_{+}$. Using the language of the algebraic geometry, we call $\varphi_{-}$a holomorphic curve in $F_{-}$. For any parabolic subgroup $P$ containing $B_{+}$, there is a natural projection from $F_{-}$onto the flag manifold $F_{P} \equiv G / P$; denote this projection $\pi_{P}$. Using such a projection, we can define a holomorphic curve $\varphi_{-P}$ in $F_{P}$ as

$$
\varphi_{-P} \equiv \pi_{-P} \circ \varphi_{-} .
$$

Thus, any holomorphic curve $\varphi_{-}$in $F_{-}$generates a family of holomorphic curves $\varphi_{-P}$ labelled by parabolic subgroups containing the Borel subgroup $B_{+}$. We are especially interested in holomorphic curves generated by the 
parabolic subgroups of the form $P_{+i^{\prime}}, i=1, \ldots, r$ (see appendix B). Denote the corresponding flag manifolds by $F_{-i}$, and the projections from $F_{-}$onto $F_{-i}$ by $\pi_{-i}$. Note that the projections $\pi_{-i}$ are defined by the relation

$$
\pi_{-i}\left(g B_{+}\right) \equiv g P_{+i^{\prime}}
$$

The flag manifolds $F_{-i}$ are closely related to the fundamental representations of the group $G$ and the corresponding representations of the Lie algebra $\mathfrak{g}$. It is well-known that for a complex semisimple Lie group of rank $r$ there are $r$ fundamental representations. Let $\rho_{i}: G \rightarrow \mathrm{GL}\left(V_{i}\right), i=1, \ldots, r$, be a family of fundamental representations of $G$. We use the notation $\rho_{i}$ also for the corresponding fundamental representation of the Lie algebra $\mathfrak{g}$. Recall that the $i$-th fundamental representation of the Lie algebra $\mathfrak{g}$ is characterised by the highest weight vector $v_{i} \in V_{i}$, satisfying the relations

$$
\rho_{i}\left(h_{j}\right) v_{i}=\delta_{i j}, \quad j=1, \ldots, r .
$$

Since $v_{i}$ is the highest weight vector, we have

$$
\rho_{i}\left(x_{+j}\right) v_{i}=0, \quad j=1, \ldots, r .
$$

Moreover, it can be shown that

$$
\rho_{i}\left(x_{-j}\right) v_{i}=0, \quad i \neq j
$$

and

$$
\rho_{i}\left(x_{-i}\right) v_{i} \neq 0 .
$$

It follows from Eqs. (4.16)-(4.19) that the line in $V_{i}$ containing the vector $v_{i}$ is invariant under the action of the parabolic subgroup $P_{+i^{\prime}}$. Note that, as any representation, the fundamental representation $\rho_{i}$ defines a left action of the group $G$ in the projective space $\mathbb{P}\left(V_{i}\right)$. Here for any $g \in G$ we have

$$
g \cdot \operatorname{pr}_{i}(v)=\operatorname{pr}_{i}\left(\rho_{i}(g) v\right), \quad v \in V_{i}^{\prime},
$$

where $\operatorname{pr}_{i}$ is the natural projection from $V_{i}^{\prime}$ onto $\mathbb{P}\left(V_{i}\right)$. Thus, we can say that the parabolic subgroup $P_{+i^{\prime}}$ is the stability subgroup of the point $p_{i} \in$ $\mathbb{P}\left(V_{i}\right)$, corresponding to the highest weight vector $v_{i}$. The above consideration 
shows that we can define an embedding $\iota_{F_{-i}}$ of the flag manifold $F_{-i}$ into the projective space $\mathbb{P}\left(V_{i}\right)$ by

$$
\iota_{F_{-i}}\left(g P_{+i^{\prime}}\right) \equiv g \cdot p_{i} .
$$

The Fubini-Study metric on $\mathbb{P}\left(V_{i}\right)$ induces a Kähler metric on $F_{-i}$; hence the flag manifold $F_{-i}$ is a Kähler manifold. The Kähler metric on $F_{-i}$, via the mapping

$$
\varphi_{-i} \equiv \pi_{-i} \circ \varphi_{-},
$$

specifies a pseudo-metric on $M$. The fundamental form $\Phi_{i}$ associated with this pseudo-metric, is given by

$$
\Phi_{i}=\left(\varphi_{-}^{*} \circ \pi_{-i}^{*} \circ \iota_{F_{-i}}^{*}\right) \Phi_{i}^{F S},
$$

where $\Phi_{i}^{F S}$ is the fundamental form associated with the Fubini-Study metric on $\mathbb{P}\left(V_{i}\right)$.

Proposition 4.1. Any holomorphic local lift $\tilde{\varphi}_{-}$of the mapping $\varphi_{-}$to $G$ leads to the following local representation of the fundamental form $\Phi_{i}$ :

$$
\Phi_{i}=-2 \sqrt{-1} \partial \bar{\partial} \ln \left\|\tilde{\varphi}-v_{i}\right\|^{2} .
$$

Proof. By definition, the mapping $\tilde{\varphi}_{-}$satisfies the relation

$$
\varphi_{-}=\pi_{-} \circ \tilde{\varphi}_{-} .
$$

Define the mapping $\tau_{i}: G \rightarrow V_{i}^{\prime}$ by

$$
\tau_{i}(g) \equiv \rho_{i}(g) v_{i}, \quad g \in G,
$$

and prove the following equality

$$
\operatorname{pr}_{i} \circ \tau_{i}=\iota_{F_{-i}} \circ \pi_{-i} \circ \pi_{-} .
$$

Indeed, using Eq. (4.20), for any $g \in G$ we get

$$
\left(\operatorname{pr}_{i} \circ \tau_{i}\right)(g)=\operatorname{pr}_{i}\left(\rho_{i}(g) v_{i}\right)=g \cdot p_{i} .
$$

From the other hand, it follows from Eqs. (4.15) and (4.21) that

$$
\left(\iota_{F_{-i}} \circ \pi_{-i} \circ \pi_{-}\right)(g)=\iota_{F_{-i}}\left(g P_{+i^{\prime}}\right)=g \cdot p_{i} .
$$


Hence, equality (4.27) is true.

So, Eqs. (4.25) and (4.26) results in

$$
\iota_{F_{-i}} \circ \pi_{-i} \circ \varphi_{-}=\operatorname{pr}_{i} \circ \tau_{i} \circ \tilde{\varphi}_{-} .
$$

Taking into account Eqs. (4.23) and (4.13), we get

$$
\Phi_{i}=\left(\tilde{\varphi}_{-}^{*} \circ \tau_{i}^{*} \circ \operatorname{pr}_{i}^{*}\right) \Phi_{i}^{F S}=-4 \sqrt{-1} \partial \bar{\partial}\left(\tilde{\varphi}_{-}^{*} \circ \tau_{i} \circ F\right) .
$$

The assertion of the proposition is the direct consequence of Eq. (4.31).

Proposition 4.1 shows that the function

$$
K_{i} \equiv \ln \left\|\tilde{\varphi}_{-} v_{i}\right\|^{2}
$$

is a Kähler potential of the pseudo-metric $g_{i}$ on $M$, having $\Phi_{i}$ as its fundamental form. Choosing different lifts $\tilde{\varphi}_{-}$, we get different Kähler potentials.

Suppose now that the hermitian scalar product in $V_{i}$ is chosen in such a way that the corresponding representation of the group $G$ is unitary. Let $\varphi_{-*}\left(\partial_{z p}\right) \in \pi_{-*}\left(\mathcal{O}_{-g}\right)$ for any $p \in M$ and $\pi_{-}(g)=p$. Consider an arbitrary local lift $\varphi$ of $\varphi_{\text {- }}$ to $G$, and construct for it a local decomposition of the form (2.60). With the help of Eq. (2.66), define the mapping $\gamma_{-}$, corresponding to the mapping $\mu_{-}$, and the mapping $\tilde{\varphi}_{-}$by

$$
\tilde{\varphi}_{-} \equiv \mu_{-} \gamma_{-}
$$

It is clear that $\tilde{\varphi}_{-}$is a holomorphic local lift of $\varphi_{-}$to $G$. It follows from theorem 3.1 that for such choice of $\tilde{\varphi}_{-}$the functions $f_{i} \equiv K_{i}$ are real solutions of the Toda equations (2.95).

Theorem 4.1. Under the conditions described above, the Ricci curvature tensors $R_{i}$ of the pseudo-metrics $g_{i}$ on $M$ are connected with the corresponding fundamental forms $\Phi_{i}$ by the relations

$$
R_{i}=\sum_{j=1}^{r} k_{i j} \Phi_{j}
$$

Proof. From Eq. (4.24) we get

$$
\Phi_{i}=-2 \sqrt{-1} \partial \bar{\partial} f_{i}
$$


where $f_{i}$ are real solutions of equations (2.95). Comparing Eqs. (4.8) and (4.10) and using equations (2.95), for the pseudo-metrics $g_{i}$ we find the expression

$$
g_{i}=2 \partial \bar{\partial} f_{i} d z d \bar{z}=4 k_{i} \exp \left[-(k f)_{i}\right] d z d \bar{z} .
$$

Taking into account Eq. (4.11) and again using equations (2.95), we have

$$
R_{i}=-2 \sqrt{-1} \sum_{j=1}^{r} k_{i j} \partial \bar{\partial} f_{j}
$$

and, with account of Eq. (4.35), we come to Eq. (4.34).

Relations (4.34) are called the generalised infinitesimal Plücker formulas. As we have already noted in the introduction, the validity of these relations was conjectured in [10], and proved in [22]. Our proof is based on the special choice of the Kähler potentials of the pseudo-metrics, and in this sense is similar to the proof of the usual Plücker formulas [14]. Note also, that the relation between the abelian Toda fields and the Kähler potentials in question, has been established for the $A$-case in [8], and for the other classical series $(B, C, D)$ - in [9], using explicit calculations in a coordinate parametrisation of the corresponding coset spaces.

In the conclusion, we would like to thank F. E. Burstall, J.-L. Gervais, B. A. Khesin, M. L. Kontsevich, Yu. I. Manin and L. M. Woodward for very useful and illuminating discussions; and $\mathrm{Ph}$. A. Griffiths for interesting communications. One of the authors (M.V.S.) is grateful to the Laboratoire de Physique Théorique de l'École Normale Supérieure de Paris for a warm hospitality during his work there in 1992 and in 1993; and the Max-Planck Institut für Mathematik in Bonn for an invitation to visit the Institute, that was very fruitful and pleasant. This work was partially supported by the ISF, grant RMO000. A initial version of the paper has appeared as a preprint LPTENS-93/45 in November 1993, and was revised in accordance with some very useful comments of a referee, to whom we are most grateful.

\section{Appendix A. Geometry of Complex Lie groups}

In this appendix we collect some information about complex Lie groups which is needed in the main text; for more details see e.g., [1, 15, 17]. 
Let us begin with the necessary definitions and facts about real Lie groups. Consider an arbitrary real Lie group $G$ with the unit element denoted $e$. Let $x \in T_{e}(G)$, define the vector field $X$ by

$$
X_{g} \equiv L_{g *}(x), \quad g \in G,
$$

where $L_{g}: h \in G \rightarrow g h \in G$ is the left translation by the element $g$. It is clear that $X$ is a left invariant vector field. The Lie bracket of any two left invariant vector fields is a left invariant vector field. The same is true for an arbitrary linear combination of left invariant vector fields; thus the space of left invariant vector fields is a Lie subalgebra of the Lie algebra of vector fields on $G$. This Lie algebra is called the Lie algebra of the Lie group $G$; we denote it $\mathfrak{g}$.

Let $X$ be a left invariant vector field on $G$; associate with it an element $x=X_{e} \in T_{e}(G)$. Such a correspondence is clearly a linear mapping. Moreover, since $X$ is left invariant, then $X_{e}=L_{g^{-1}}\left(X_{g}\right)$ for any $g \in G$. Hence, we see that different left invariant vector fields correspond to different elements of $T_{e}(G)$, and we have an isomorphism of linear spaces. This isomorphism becomes an isomorphism of Lie algebras if we introduce in $T_{e}(G)$ the structure of a Lie algebra by

$$
[x, y] \equiv[X, Y]_{e}, \quad x, y \in T_{e}(G),
$$

where $X$ and $Y$ are left invariant vector fields defined with the help of Eq. (A.1). Thus we can identify the Lie algebra $\mathfrak{g}$ with $T_{e}(G)$. Below we use the latter interpretation of the Lie algebra of a Lie group.

The adjoint representation $\operatorname{Ad}: g \in G \rightarrow \operatorname{Ad}(g) \in \operatorname{GL}(\mathfrak{g})$ of the group $G$ is defined by the relation

$$
\operatorname{Ad}(g) x \equiv\left(L_{g} \circ R_{g^{-1}}\right)_{*}(x), \quad g \in G,
$$

where $R_{g}: h \in G \rightarrow h g \in G$ is the right translation by the element $g$. The corresponding adjoint representation ad $: x \in \mathfrak{g} \rightarrow \operatorname{ad}(x) \in \mathrm{GL}(\mathfrak{g})$ of the Lie algebra $\mathfrak{g}$ is given by

$$
\operatorname{ad}(x) y \equiv[x, y], \quad x, y \in \mathfrak{g} .
$$


The Maurer-Cartan form of $G$ is a 1 -form $\theta$ on $G$ taking values in $\mathfrak{g}$ and defined by the relation

$$
\theta(x) \equiv L_{g^{-1} *}(x)
$$

for any $x \in T_{e}(G)$. The Maurer-Cartan form is left invariant, i.e.,

$$
L_{g *} \theta=\theta
$$

for any $g \in G$. The transformation of $\theta$ under the right translations is described by the formula

$$
R_{g *} \theta=\operatorname{Ad}\left(g^{-1}\right) \circ \theta .
$$

It can be also shown that

$$
d \theta(X, Y)+[\theta(X), \theta(Y)]=0
$$

for any vector fields $X$ and $Y$ on $G$.

Let $\Sigma$ be a homomorphism of the group $G$, and

$$
\sigma \equiv \Sigma_{* e}
$$

be the corresponding homomorphism of $\mathfrak{g}$. It follows from the definition of the adjoint representation of $G$ that

$$
\sigma \circ \operatorname{Ad}(g)=\operatorname{Ad}(\Sigma(g)) \circ \sigma .
$$

Furthermore, it can be easily shown that

$$
\Sigma^{*} \theta=\sigma \circ \theta
$$

In this paper we mainly deal with complex differentiable manifolds. Recall some relevant definitions and introduce notations. Let $V$ be a complex linear space. On the space $V_{\mathbb{R}}$ there is a natural complex structure $J^{V}$ generated by the multiplication by $\sqrt{-1}$ in $V$. Consider the space $\left(V_{\mathbb{R}}\right)^{\mathbb{C}}$. The linear space $V_{\mathbb{R}}$ can be naturally considered as a real subspace of $\left(V_{\mathbb{R}}\right)^{\mathbb{C}}$. Any element $v$ of $\left(V_{\mathbb{R}}\right)^{\mathbb{C}}$ has a unique representation of the form

$$
v=u+\sqrt{-1} w
$$


where $u, w \in V_{\mathbb{R}}$. The complex conjugation in $\left(V_{\mathbb{R}}\right)^{\mathbb{C}}$ is defined by

$$
\bar{v}=u-\sqrt{-1} w
$$

where $u, w$ are elements of $V_{\mathbb{R}}$ entering representation (A.12).

Let $W$ be another complex linear space, and $\varphi$ be a linear mapping from $\left(V_{\mathbb{R}}\right)^{\mathbb{C}}$ to $\left(W_{\mathbb{R}}\right)^{\mathbb{C}}$. The complex conjugate mapping $\bar{\varphi}$ acts on the elements of $\left(V_{\mathbb{R}}\right)^{\mathbb{C}}$ according to the rule

$$
\bar{\varphi} v \equiv \bar{\varphi} \bar{v}, \quad v \in\left(V_{\mathbb{R}}\right)^{\mathbb{C}}
$$

The mapping $\varphi$ is called real if $\bar{\varphi}=\varphi$.

The operator $J^{V}$ can be naturally extended to the linear operator on $\left(V_{\mathbb{R}}\right)^{\mathbb{C}}$, which will be also denoted $J^{V}$. Define the subspaces $V^{(1,0)}$ and $V^{(0,1)}$ of the space $\left(V_{\mathbb{R}}\right)^{\mathbb{C}}$ by

$$
\begin{aligned}
& V^{(1,0)}=\left\{v \in\left(V_{\mathbb{R}}\right)^{\mathbb{C}} \mid J^{V} v=\sqrt{-1} v\right\} \\
& V^{(0,1)}=\left\{v \in\left(V_{\mathbb{R}}\right)^{\mathbb{C}} \mid J^{V} v=-\sqrt{-1} v\right\} .
\end{aligned}
$$

The space $\left(V_{\mathbb{R}}\right)^{\mathbb{C}}$ is a direct sum of the subspaces $V^{(1,0)}$ and $V^{(0,1)}$. Hence, any element $v \in\left(V_{\mathbb{R}}\right)^{\mathbb{C}}$ can be uniquely written in the form

$$
v=v^{(1,0)}+v^{(0,1)}
$$

where $v^{(1,0)} \in V^{(1,0)}$ and $v^{(0,1)} \in V^{(0,1)}$. The linear operators

$$
P^{V} \equiv \frac{1}{2}\left(I-\sqrt{-1} J^{V}\right), \quad \bar{P}^{V} \equiv \frac{1}{2}\left(I+\sqrt{-1} J^{V}\right) .
$$

project $\left(V_{\mathbb{R}}\right)^{\mathbb{C}}$ on the subspaces $V^{(1,0)}$ and $V^{(0,1)}$, respectively. Note that $P^{V}$ and $\bar{P}^{V}$ are connected by the complex conjugation, that is already reflected in their notation.

Let $M$ be a complex manifold. The natural complex structure on $M$ will be denoted $J^{M}$; by $T_{\mathbb{R} p}(M)$ we denote the usual real tangent space to $M$ at the point $p$, where $M$ is considered as a real manifold; and the complexification of $T_{\mathbb{R} p}(M)$ is denoted by $T_{p}^{\mathbb{C}}(M)$. As above, we have the decomposition of $T_{p}^{\mathbb{C}}(M)$ into a direct sum

$$
T_{p}^{\mathbb{C}}(M)=T^{(1,0)}(M) \oplus T^{(0,1)}(M)
$$


Let $P_{p}^{M}$ be the mapping projecting an element of $T_{p}^{\mathbb{C}}(M)$ to its $(1,0)$-component, in other words

$$
P_{p}^{M} x \equiv \frac{1}{2}\left(x-\sqrt{-1} J_{p}^{M} x\right)
$$

for any vector $x \in T_{p}^{\mathbb{C}}(M)$. The mapping $P^{M}$ projecting a vector field $X$ on $M$ to its $(1,0)$-component is defined by

$$
\left(P^{M} X\right)_{p} \equiv P_{p}^{M} X_{p}
$$

for any vector field $X$ on $M$. The projection mappings to the $(0,1)$-component of a tangent vector and a vector field are defined similarly.

Now, let $G$ be a complex Lie group. In this case the group operation $(g, h) \in$ $G \times G \rightarrow g h^{-1} \in G$ is, by definition, a holomorphic mapping. The group $G$ can be considered as a real Lie group supplied with a complex structure $J^{G}$. Denote the corresponding real Lie algebra $\mathfrak{g}_{\mathbb{R}}$. Since the group operation in $G$ is holomorphic, we have

$$
\begin{aligned}
& L_{g *} \circ J^{G}=J^{G} \circ L_{g *}, \\
& R_{g_{*}} \circ J^{G}=J^{G} \circ R_{g *} .
\end{aligned}
$$

The restriction of the complex structure $J^{G}$ to $T_{e}(G)$ generates a complex structure $J^{\mathfrak{g}_{\mathbb{R}}}$ on the Lie algebra $\mathfrak{g}_{\mathbb{R}}$. It follows from Eqs. (A.22) and (A.23) that

$$
\operatorname{ad}(x) \circ J^{\mathfrak{g}_{\mathbb{R}}}=J^{\mathfrak{g}_{\mathbb{R}}} \circ \operatorname{ad}(x)
$$

for any $x \in \mathfrak{g}_{\mathbb{R}}$. Hence, $J^{\mathfrak{g}_{\mathbb{R}}}$ is a Lie complex structure, and $\mathfrak{g}_{\mathbb{R}}$ has the structure of a complex Lie algebra; denote it $\mathfrak{g}$. Actually the real Lie algebra $\mathfrak{g}_{\mathbb{R}}$ is the realification of the complex Lie algebra $\mathfrak{g}$, that is already reflected in the notation $\mathfrak{g}_{\mathbb{R}}$. The (complex) Lie algebra $\mathfrak{g}$ is called the Lie algebra of the (complex) Lie group $G$. In fact, there exists another interpretation of the Lie algebra of a complex Lie group, that is more convenient for our purposes. To give this interpretation let us recall some facts from the theory of Lie algebras.

Let $\mathfrak{g}$ be a complex Lie algebra. Consider the space $\left(\mathfrak{g}_{\mathbb{R}}\right)^{\mathbb{C}}$. This space has a natural structure of a complex Lie algebra. It can be shown that the 
decomposition

$$
\left(\mathfrak{g}_{\mathbb{R}}\right)^{\mathbb{C}}=\mathfrak{g}^{(1,0)} \oplus \mathfrak{g}^{(0,1)} .
$$

is a decomposition of $\left(\mathfrak{g}_{\mathbb{R}}\right)^{\mathbb{C}}$ into a direct sum of two Lie subalgebras. Moreover, we have

$$
\begin{array}{lll}
\text { (A.26) } & P^{\mathfrak{g}}\left(a x+b J^{\mathfrak{g}_{\mathfrak{R}}} x\right)=(a+\sqrt{-1} b) P^{\mathfrak{g}} x, & P^{\mathfrak{g}}[x, y]=\left[P^{\mathfrak{g}} x, P^{\mathfrak{g}} y\right], \\
\text { (A.27) } & \bar{P}^{\mathfrak{g}}\left(a x-b J^{\mathfrak{g}_{\mathfrak{R}}} x\right)=(a+\sqrt{-1} b) \bar{P}^{\mathfrak{g}} x, & \bar{P}^{\mathfrak{g}}[x, y]=\left[\bar{P}^{\mathfrak{g}} x, \bar{P}^{\mathfrak{g}} y\right]
\end{array}
$$

for all $a, b \in \mathbb{R}$ and $x, y \in\left(\mathfrak{g}_{\mathbb{R}}\right)^{\mathbb{C}}$. Restricting $P^{\mathfrak{g}}$ and $\bar{P}^{\mathfrak{g}}$ to $\mathfrak{g}_{\mathbb{R}}$, we get bijective mappings from $\mathfrak{g}_{\mathbb{R}}$ to $\mathfrak{g}^{(1,0)}$ and $\mathfrak{g}^{(0,1)}$. Taking into account Eqs. (A.26) and (A.27), we see that the first mapping sets an isomorphism between the Lie algebras $\mathfrak{g}$ and $\mathfrak{g}^{(1,0)}$, while the second one sets an isomorphism between the Lie algebras $\overline{\mathfrak{g}}$ and $\mathfrak{g}^{(0,1)}$. Here $\overline{\mathfrak{g}}$ is a complex Lie algebra which is obtained from $\mathfrak{g}_{\mathbb{R}}$ with the help of the Lie complex structure $-J^{\mathfrak{g} \text { p. }}$.

Thus, we can identify the Lie algebra of the Lie group $G$ with the holomorphic tangent space $T_{e}^{(1,0)}(G) \subset T_{e}^{\mathbb{C}}(G)$. Actually, any element of the space $T_{e}^{(1,0)}(G)$ generates a holomorphic left invariant vector field on $G$ given by the relation of form (A.1); and the Lie algebra operation in $\mathfrak{g}$ is related to the Lie bracket of the corresponding holomorphic left invariant vector fields by Eq. (A.2). Note that any left invariant vector field on $G$ of the type $(1,0)$ is a holomorphic vector field.

Considering $G$ as a real Lie group, we can define on it the corresponding Maurer-Cartan form $\theta_{\mathbb{R}}$ taking values in $\mathfrak{g}_{\mathbb{R}}$. It is clear that

$$
\theta_{\mathbb{R}}\left(J^{G}(X)\right)=J_{e}^{G} \circ \theta_{\mathbb{R}}(X)
$$

for any vector field $X$ on $G_{\mathbb{R}}$. The complexification $\left(\theta_{\mathbb{R}}\right)^{\mathbb{C}}$ takes values in $\left(\mathfrak{g}_{\mathbb{R}}\right)^{\mathbb{C}}$. The Maurer-Cartan form $\theta$ of the complex Lie group $G$ is defined by

$$
\theta=P_{e}^{G} \circ\left(\theta_{\mathbb{R}}\right)^{\mathbb{C}} .
$$

It follows from this definition that $\theta$ takes values in the Lie algebra $\mathfrak{g}$. Using Eq. (A.28), we get

$$
\theta(X)=\left(\theta_{\mathbb{R}}\right)^{\mathbb{C}}\left(P^{G} X\right)
$$


hence, $\theta$ is a 1 -form of the type $(1,0)$. Moreover, it can be shown that it is a holomorphic 1-form.

Actually, we can define $\theta$ as the unique 1-form of the type $(1,0)$, satisfying relation (A.5), where $X$ now is an arbitrary left invariant vector field on $G$ of the type $(1,0)$. It can be shown that in the case of a complex Lie group we still have relations (A.6)-(A.8). The situation with relation (A.11) is more complicated. In fact, it is natural to consider only holomorphic and antiholomorphic automorphisms of $G$. For a holomorphic automorphism $\Sigma$ we have that Eq. (A.11) is valid with $\sigma$ defined by Eq. (A.9). If $\Sigma$ is an antiholomorphic automorphism of $G$, then

$$
\Sigma_{* e} T_{e}^{(1,0)}(G)=T_{e}^{(0,1)}(G)
$$

In this case we define $\sigma$ as an antilinear automorphism of $\mathfrak{g}$ by

$$
\sigma x \equiv \overline{\Sigma_{* e}(x)} \quad x \in \mathfrak{g} .
$$

This definition leads to the equality

$$
\Sigma^{*} \theta(x)=(\sigma \circ \theta)(\bar{x}),
$$

which is valid for any $x \in T_{g}^{\mathbb{C}}(G), g \in G$. Note that relation (A.10) is valid for both cases with the mapping $\sigma$ defined either by Eq.(A.9), or by Eq.(A.32).

\section{Appendix B. Complex Lie Algebras, Flag Manifolds, and $\mathbb{Z}$-GRAdATIONS}

In this appendix we present some facts on complex semisimple finite dimensional Lie algebras, see e.g. $[1,12,15,11]$, needed in the main text. We also consider here the concept of a flag manifold and its relation to the concept of $\mathbb{Z}$-gradation of a Lie algebra.

Let $\mathfrak{g}$ be a complex semisimple Lie algebra, and $\mathfrak{h}$ be a fixed Cartan subalgebra of $\mathfrak{g}$. Further, let $\Delta$ be the system of the nonzero roots of $\mathfrak{g}$ with respect to $\mathfrak{h}$. The root subspaces $\mathfrak{g}^{\alpha}, \alpha \in \Delta$, are defined as

$$
\mathfrak{g}^{\alpha} \equiv\{x \in \mathfrak{g} \mid[h, x]=\alpha(h) x \text { for all } h \in \mathfrak{h}\}
$$


All the root subspaces are one-dimensional, and the Lie algebra $\mathfrak{g}$ is represented as a direct sum

$$
\mathfrak{g}=\mathfrak{h} \oplus \bigoplus_{\alpha \in \Delta} \mathfrak{g}^{\alpha} .
$$

Choose in each subspace $\mathfrak{g}^{\alpha}$ a nonzero vector $x_{\alpha}$, then

$$
\left[x_{\alpha}, x_{\beta}\right]=n_{\alpha, \beta} x_{\alpha+\beta}
$$

if $\alpha+\beta \in \Delta$, while

$$
\left[x_{\alpha}, x_{\beta}\right]=0
$$

if $\alpha+\beta \notin \Delta$ and $\alpha+\beta \neq 0$.

Let ( , ) be the Killing-Cartan form of $\mathfrak{g}$. Define the vectors $h_{\alpha} \in \mathfrak{h}, \alpha \in \Delta$, by

$$
\left(h, h_{\alpha}\right)=\alpha(h), \quad h \in \mathfrak{h} .
$$

It can be shown that the vectors $x_{\alpha}$ can be chosen in such a way that

$$
\left[x_{\alpha}, x_{-\alpha}\right]=h_{\alpha}
$$

and the constants $n_{\alpha, \beta}$ satisfy the relation

$$
n_{\alpha, \beta}=-n_{-\alpha,-\beta}
$$

In this case $n_{\alpha, \beta}$ are real numbers. The set of the $x_{\alpha}, \alpha \in \Delta$, having the properties described above, is called the Weyl basis of $\mathfrak{g}$ modulo $\mathfrak{h}$.

Recall that any involutive antilinear automorphism $\sigma$ of $\mathfrak{g}$ specifies a real form $\mathfrak{g}_{\sigma}$ of $\mathfrak{g}$ defined by

$$
\mathfrak{g}_{\sigma} \equiv\{x \in \mathfrak{g} \mid \sigma(x)=x\} .
$$

Using the Weyl basis of $\mathfrak{g}$, we can define an antilinear automorphism $\sigma$ of $\mathfrak{g}$ with the help of the relations

$$
\sigma\left(h_{\alpha}\right)=-h_{\alpha}, \quad \sigma\left(x_{\alpha}\right)=-x_{-\alpha} .
$$

The corresponding real form $\mathfrak{u}$ in this case is compact.

Let $\Delta=\Delta_{-} \cup \Delta_{+}$be a representation of the root system of $\mathfrak{g}$ as a disjoint union of the positive and negative roots with respect to some lexicographic 
ordering, and $\Pi \subset \Delta_{+}$be a system of simple roots. Denote the elements of $\Pi$ by $\alpha_{i}, i=1, \ldots, r \equiv \operatorname{rank} \mathfrak{g}$. Let $\left\{x_{\alpha}\right\}$ be the Weyl basis of $\mathfrak{g}$. The elements

$$
h_{i} \equiv \frac{2}{\left(\alpha_{i}, \alpha_{i}\right)} h_{\alpha_{i}} \quad x_{ \pm i} \equiv x_{ \pm \alpha_{i}}
$$

generate the whole Lie algebra $\mathfrak{g}$. Under an appropriate normalisation, the elements $x_{ \pm i}$, together with the Cartan generators $h_{i}$, satisfy the defining relations

$$
\left[h_{i}, h_{j}\right]=0, \quad\left[h_{i}, x_{ \pm j}\right]= \pm k_{j i} x_{ \pm j}, \quad\left[x_{+i}, x_{-j}\right]=\delta_{i j} h_{i},
$$

where $k$ is the Cartan matrix of $\mathfrak{g}$. The elements $x_{ \pm i}$ are called Chevalley generators of $\mathfrak{g}$.

A Lie subalgebra $\mathfrak{b}$ is called a Borel subalgebra of $\mathfrak{g}$ if $\mathfrak{b}$ is a maximal solvable subalgebra of $\mathfrak{g}$. Denote by $G$ a complex connected Lie group having $\mathfrak{g}$ as its Lie algebra. A subgroup $B$ of $G$ is called a Borel subgroup if its Lie algebra $\mathfrak{b}$ is a Borel subalgebra of $\mathfrak{g}$. According to the Borel-Morozov theorem, all Borel subalgebras of $\mathfrak{g}$ are connected one to each other by the action of the group Aut $\mathfrak{g}$ of automorphisms of $\mathfrak{g}$. The similar statement is valid also for the Borel subgroups of $G$.

The root decomposition (B.2) of $\mathfrak{g}$ implies that

$$
\mathfrak{g}=\mathfrak{n}_{-} \oplus \mathfrak{h} \oplus \mathfrak{n}_{+}
$$

where

$$
\mathfrak{n}_{ \pm} \equiv \bigoplus_{\alpha \in \Delta_{ \pm}} \mathfrak{g}^{\alpha}
$$

are nilpotent subalgebras of $\mathfrak{g}$. The corresponding connected nilpotent Lie subgroups of $G$ will be denoted $N_{ \pm}$. It can be shown that the subalgebras

$$
\mathfrak{b}_{ \pm} \equiv \mathfrak{h} \oplus \mathfrak{n}_{ \pm}
$$

are Borel subalgebras of $\mathfrak{g}$. The Borel subalgebra $\mathfrak{b}_{-}$is called opposite to the Borel subalgebra $\mathfrak{b}_{+}$. The subalgebras $\mathfrak{b}_{ \pm}$generate Borel subgroups denoted $B_{ \pm}$.

A parabolic subalgebra of $\mathfrak{g}$ is, by definition, a subalgebra of $\mathfrak{g}$ which contains a Borel subalgebra of $\mathfrak{g}$. A subgroup $P$ of the Lie group $G$ is called a 
parabolic subgroup if it contains a Borel subgroup of $G$. Any subsystem $\Psi$ of the system of simple roots $\Pi$ generates parabolic subalgebras

$$
\mathfrak{p}_{ \pm \Psi} \equiv \mathfrak{b}_{ \pm} \oplus \bigoplus_{\alpha \in[\Psi] \cap \Sigma_{\mp}} \mathfrak{g}^{\alpha}
$$

where $[\Psi]$ is the set of all roots from $\Delta$ which can be represented as linear combinations of the roots from $\Psi$. It can be shown that, up to transformations of the group Aut $\mathfrak{g}$, any parabolic subalgebra of $\mathfrak{g}$ can be obtained in such a way. Thus, we have a transparent classification of the parabolic subalgebras of any complex semisimple Lie algebra. One usually writes $\mathfrak{p}_{ \pm\left\{i_{1}, \ldots, i_{k}\right\}}$ for the parabolic subalgebra corresponding to the subsystem $\Psi=\left\{\alpha_{i_{1}}, \ldots, \alpha_{i_{k}}\right\}$. The parabolic subalgebras corresponding to a subsystem which consists of just one simple root $\alpha_{i}$, is denoted $\mathfrak{p}_{ \pm i}$. At last, the parabolic subalgebra corresponding to a subsystem consisting of all simple roots except $\alpha_{i}$, is denoted $\mathfrak{p}_{ \pm i^{\prime}}$.

Let $P$ be a parabolic subgroup of $G$. The homogeneous space $F \equiv G / P$ is called a flag manifold or, quite rarely, a parabolic space. It is clear that $F$ has the natural structure of a complex manifold. Let $\mathfrak{u}$ be a compact form of $\mathfrak{g}$ defined by the antilinear involutive automorphism $\sigma$ given by relations (B.9), and $\mathfrak{a}$ be a maximal abelian subalgebra of $\mathfrak{u}$. Denote by $U$ and $A$ the corresponding real connected Lie groups. According to the Borel-Hirzebruch theorem, the flag manifolds $G / B_{ \pm}$are diffeomorphic to the homogeneous space $U / A$. It follows from this fact that for any Borel subgroup $B$, the flag manifold $G / B$ is compact. Since any parabolic subgroup $P$ contains some Borel subgroup $B$, there is the natural projection from $G / B$ to $G / P$; hence all flag manifolds are compact.

Consider the realification $\mathfrak{g}_{\mathbb{R}}$ of the Lie algebra $\mathfrak{g}$. Let $J^{\mathfrak{g}_{\mathbb{R}}}$ be the linear operator in $\mathfrak{g}_{\mathbb{R}}$, corresponding to the multiplication by $\sqrt{-1}$ in $\mathfrak{g}$. The expansion of $\mathfrak{g}_{\mathbb{R}}$ into the direct sum

$$
\mathfrak{g}_{\mathbb{R}}=\mathfrak{u} \oplus J^{\mathfrak{g} \mathbb{R}} \mathfrak{a} \oplus \mathfrak{n}_{+\mathbb{R}}
$$

where subalgebras $\mathfrak{u}, \mathfrak{a}$ and $\mathfrak{n}_{+}$were defined above, is called the Iwasawa decomposition of $\mathfrak{g}$. Note that

$$
\mathfrak{h}_{\mathbb{R}}=\mathfrak{a} \oplus J^{\mathfrak{g}_{\mathbb{R}}} \mathfrak{a} .
$$


There is the corresponding analytic decomposition of the Lie group $G$ considered as a real Lie group. It has the form

$$
G=U N_{+} A^{*},
$$

where $A^{*}$ is the real connected Lie group corresponding to the subalgebra $J^{\mathfrak{g} p} \mathfrak{a}$.

A decomposition of $\mathfrak{g}$ into a direct sum of subspaces $\mathfrak{g}_{m}$,

$$
\mathfrak{g}=\bigoplus_{m \in \mathbb{Z}} \mathfrak{g}_{m}
$$

such that

$$
\left[\mathfrak{g}_{m}, \mathfrak{g}_{k}\right] \subset \mathfrak{g}_{m+k},
$$

is called a $\mathbb{Z}$-gradation of $\mathfrak{g}$. For any $\mathbb{Z}$-gradation of $\mathfrak{g}$ we can construct a homomorphism $\rho: \mathbb{C}^{*} \rightarrow$ Aut $\mathfrak{g}$, where $\mathbb{C}^{*} \equiv \mathbb{C}-\{0\}$ is considered as a complex Lie group with respect to the multiplication. The homomorphism $\rho$ is defined by

$$
\rho(\lambda) x=\rho(\lambda)\left(\sum_{m \in \mathbb{Z}} x_{m}\right) \equiv \sum_{m \in \mathbb{Z}} \lambda^{m} x_{m}, \quad \lambda \in \mathbb{C}^{*} .
$$

Actually, the mapping $\rho$ takes values in the connected component of the group Aut $\mathfrak{g}$ containing unity, which, for the considered case of a semisimple Lie algebra, coincides with the Lie group Int $\mathfrak{g}$ of internal automorphisms of $\mathfrak{g}$. The Lie algebra of the group Int $\mathfrak{g}$ is the Lie algebra ad $\mathfrak{g}$. Hence, there exists an element $q$ of $\mathfrak{g}$ such that

$$
\left[q, x_{m}\right]=m x_{m}
$$

for any $x_{m} \in \mathfrak{g}_{m}$. The element $q$ is called the gradation operator of a given $\mathbb{Z}$-gradation. Thus, for a complex semisimple Lie algebra, any $\mathbb{Z}$-gradation may be defined with the help of the corresponding gradation operator.

It is clear that the linear operator $\operatorname{ad}(q)$ is semisimple and satisfies the relation

$$
\exp (2 \pi i \operatorname{ad}(q))=\mathrm{id}_{\mathfrak{g}} .
$$

Since we consider the case of a semisimple Lie algebra, the element $q$ is semisimple. From the other hand, it is clear that any semisimple element 
$q$ of $\mathfrak{g}$ satisfying Eq. (B.23), can be considered as the gradation operator of some $\mathbb{Z}$-gradation of $\mathfrak{g}$.

Since the element $q$ is semisimple, without any loss of generality we can suppose that $q \in \mathfrak{h}$ and $\alpha_{i}(q) \geq 0, i=1, \ldots, r$. It follows from Eq. (B.23) that for any $i$, the number $n_{i} \equiv \alpha_{i}(q)$ is an integer. The element $q$ is uniquely defined by the numbers $n_{i}$. Indeed, from Eq. (B.11) we have

$$
q=\sum_{i, j=1}^{r} k_{i j}^{-1} n_{j} h_{i}
$$

Note that the subspace $\mathfrak{g}_{m}$ is the sum of the root spaces $\mathfrak{g}_{\alpha}$, corresponding to the roots $\alpha=\sum_{1 \leq i \leq r} c_{i} \alpha_{i}$ with $\sum_{1 \leq i \leq r} c_{i} n_{i}=m$. The subspace $\mathfrak{g}_{0}$ includes also the Cartan subalgebra $\mathfrak{h}$. Any positive root belongs to some subspace $\mathfrak{g}_{m}$ with $m \geq 0$, while any negative root belongs to some subspace $\mathfrak{g}_{m}$ with $m \leq 0$. Further, if $\mathfrak{g}^{\alpha} \subset \mathfrak{g}_{m}$, then $\mathfrak{g}^{-\alpha} \subset \mathfrak{g}_{-m}$; hence, we have

$$
\operatorname{dim} \mathfrak{g}_{m}=\operatorname{dim} \mathfrak{g}_{-m} .
$$

The subspaces $\tilde{\mathfrak{n}}_{ \pm}$, defined by

$$
\tilde{\mathfrak{n}}_{-} \equiv \bigoplus_{m<0} \mathfrak{g}_{m}, \quad \tilde{\mathfrak{n}}_{+} \equiv \bigoplus_{m>0} \mathfrak{g}_{m}
$$

are nilpotent subalgebras of $\mathfrak{g}$. It is clear that

$$
\tilde{\mathfrak{n}}_{ \pm} \subset \mathfrak{n}_{ \pm}
$$

Introduce the notation

$$
\tilde{\mathfrak{b}}_{-} \equiv \bigoplus_{m \leq 0} \mathfrak{g}_{m}, \quad \tilde{\mathfrak{b}}_{+} \equiv \bigoplus_{m \geq 0} \mathfrak{g}_{m}
$$

Since $\mathfrak{h} \subset \mathfrak{g}_{0}$, we conclude that $\tilde{\mathfrak{b}}_{ \pm} \supset \mathfrak{b}_{ \pm}$. Hence $\tilde{\mathfrak{b}}_{ \pm}$are parabolic subalgebras of $\mathfrak{g}$. Defining the set $\Pi_{0} \subset \Pi$ by

$$
\Pi_{0} \equiv\left\{\alpha_{i} \in \Pi \mid \alpha_{i}(q)=0\right\},
$$

we can write

$$
\tilde{\mathfrak{b}}_{ \pm}=\mathfrak{p}_{ \pm \Pi_{0}} .
$$


From the other hand, let a parabolic subalgebra $\mathfrak{p}_{\Psi}$ with $\Psi$ being a subsystem of $\Pi$, be given. Consider an element $q \in \mathfrak{h}$, such that $\alpha_{i}(q)=0$ if and only if $\alpha_{i} \in \Psi$, and all numbers $\alpha_{i}(q)$ are nonnegative integers. It is clear that the element $q$ is the gradation operator for a $\mathbb{Z}$-gradation of $\mathfrak{g}$ with

$$
\tilde{\mathfrak{b}}_{ \pm}=\mathfrak{p}_{ \pm \Psi}
$$

Thus, for any parabolic subalgebra $\mathfrak{p}$ of $\mathfrak{g}$ we can find a $\mathbb{Z}$-gradation of $\mathfrak{g}$, such that the corresponding subalgebra $\tilde{\mathfrak{p}}_{+}$(or $\tilde{\mathfrak{p}}_{-}$) is connected with $\mathfrak{p}$ by a transformation of the group Aut $\mathfrak{g}$.

It is natural to denote the subalgebra $\mathfrak{g}_{0}$ by $\tilde{\mathfrak{h}}$. Recall that $\tilde{\mathfrak{h}}$ is not, in general, a Cartan subalgebra of $\mathfrak{g}$, but it contains some such subalgebra. Using the notations introduced above, we can write

$$
\tilde{\mathfrak{b}}_{ \pm}=\tilde{\mathfrak{h}} \oplus \tilde{\mathfrak{n}}_{ \pm}
$$

and

$$
\mathfrak{g}=\tilde{\mathfrak{n}}_{-} \oplus \tilde{\mathfrak{h}} \oplus \tilde{\mathfrak{n}}_{+} .
$$

For any complex semisimple Lie algebra there is one distinguished $\mathbb{Z}$-gradation, arising when we choose all the numbers $n_{i}$ equal to 1 . The corresponding gradation operator have in this case the form

$$
q=\sum_{i=1}^{r} k_{i} h_{i}
$$

where

$$
k_{i} \equiv \sum_{j=1}^{r} k_{i j}^{-1} .
$$

Such a gradation is called the canonical gradation. For the canonical gradation all the quantities with tildes defined above are equal to the corresponding quantities without tildes; and, of course, the subalgebra $\mathfrak{g}_{0}$ is abelian; it is a Cartan subalgebra. Note also that in this case the subspaces $\mathfrak{g}_{ \pm 1}$ coincide with the linear spans of the vectors $x_{ \pm i}, i=1, \ldots, r$.

It is convenient for our purposes to consider $\mathbb{Z}$-gradations of $\mathfrak{g}$ related to the embeddings of the Lie algebra $\mathfrak{s l}(2, \mathbb{C})$ into $\mathfrak{g}$. Recall that the Lie algebra 
$\mathfrak{s l}(2, \mathbb{C})$ consists of all traceless $2 \times 2$ matrices. The matrices

$$
x_{-} \equiv\left(\begin{array}{ll}
0 & 0 \\
1 & 0
\end{array}\right), \quad h \equiv\left(\begin{array}{rr}
1 & 0 \\
0 & -1
\end{array}\right), \quad x_{+} \equiv\left(\begin{array}{ll}
0 & 1 \\
0 & 0
\end{array}\right)
$$

form a basis in $\mathfrak{s l}(2, \mathbb{C})$ called the canonical basis. For these matrices we have

$$
\left[h, x_{ \pm}\right]= \pm 2 x_{ \pm}, \quad\left[x_{+}, x_{-}\right]=h .
$$

By an embedding of $\mathfrak{s l}(2, \mathbb{C})$ into $\mathfrak{g}$ we mean a nontrivial homomorphism from $\mathfrak{s l}(2, \mathbb{C})$ into $\mathfrak{g}$; their complete classification has been done by E. B. Dynkin. The images of the elements $h$ and $x_{ \pm}$under such a homomorphism are denoted usually by the same letters. From the properties of the representations of the Lie algebra $\mathfrak{s l}(2, \mathbb{C})$ it follows, that the element $h$ of $\mathfrak{g}$ should be semisimple, and the elements $x_{ \pm} \in \mathfrak{g}$ should be nilpotent. Moreover, it is clear that

$$
\exp (2 \pi i \operatorname{ad}(h))=\mathrm{id}_{\mathfrak{g}} .
$$

As above, without any loose of generality, one can suppose that $h \in \mathfrak{h}$, and $\alpha_{i}(h) \geq 0$ for all $\alpha_{i} \in \Pi$. It can be shown that the numbers $\alpha_{i}(h)$ are equal only to 0,1 and 2. So, the element $h$ can be considered as the gradation operator of some $\mathbb{Z}$-gradation of $\mathfrak{h}$. In fact, it is more convenient for our purposes to define the gradation operator $q$, connected with the given embedding of $\mathfrak{s l}(2, \mathbb{C})$ into $\mathfrak{g}$, by the relation

$$
h=2 q .
$$

It is clear that this definition leads to the necessity to consider also $\mathbb{Z} / 2$ gradations of $\mathfrak{g}$. If, instead of Eq. (B.38), we have the relation

$$
\exp (\pi i \operatorname{ad}(h))=\mathrm{id}_{\mathfrak{g}},
$$

we call the corresponding embedding integral, otherwise we deal with a semiintegral embedding. For an integral embedding the numbers $\alpha_{i}(h)$ are equal to 0 or 2 .

The properties of the representations of the algebra $\mathfrak{s l}(2, \mathbb{C})$ imply that if we consider a $\mathbb{Z}$-gradation, or a $\mathbb{Z} / 2$-gradation, associated with an embedding of $\mathfrak{s l}(2, \mathbb{C})$ into $\mathfrak{g}$, then

$$
\operatorname{dim} \mathfrak{g}_{0} \geq \operatorname{dim} \mathfrak{g}_{ \pm 1}
$$


For any complex semisimlpe Lie algebra there is an embedding of $\mathfrak{s l}(2, \mathbb{C})$ leading to the canonical gradation. This embedding is defined by

$$
x_{ \pm}=\sum_{i=1}^{r}\left(2 k_{i}\right)^{1 / 2} x_{ \pm i}, \quad h=2 \sum_{i=1}^{r} k_{i} h_{i}
$$

with $k_{i}$, given by Eq. (B.35), and is called the principal embedding; its exhaustive investigation has been done by B. Kostant.

It is known that not any element $h \in \mathfrak{h}$, even satisfying the requirement $\alpha_{i}(h)=0,1$ or 2 , can be considered as the corresponding element of some embedding of $\mathfrak{s l}(2, \mathbb{C})$ into $\mathfrak{g}$, and we have not here a direct relation to parabolic subalgebras, as it was for the case of a general $\mathbb{Z}$-gradation.

\section{REFERENCES}

1. Bourbaki, N., Groupes et algèbres de Lie, Ch.1. Hermann, Paris, 1960; Ch. 2 et 3. Hermann, Paris, 1972; Ch. 4, 5 et 6 . Hermann, Paris, 1968; Ch. 7 et 8 . Hermann, Paris, 1975; Ch. 9. Masson, Paris, 1982.

2. Bolton, J., Pedit, F. and Woodward, L. M., Minimal surfaces and the affine Toda field model, to appear in J. für die Reine und Angew. Math.

3. Bolton, J. and Woodward, L. M., The affine Toda equations and minimal surfaces, Harmonic maps and integrable systems (A. P. Fordy and J. C. Wood, eds.), pp. 59-82, Vieweg, 1993.

4. Burstall, F. E., Harmonic maps and soliton theory, Matem. Contemp. 2 (1992), 1-18.

5. Burstall, F. E., Ferus, D., Pedit, F. and Pinkall, U., Harmonic tori in symmetrical spaces and commuting Hamiltonian systems on loop algebras, Ann. Math. 138 (1993), 173-212.

6. Burstall, F. E. and Rawnsley, J. H., Twistor theory for Riemannian symmetric spaces, Lect. Notes Math., Vol. 1424, Springer-Verlag, Berlin, Heidelberg, 1990.

7. Gervais, J.-L. and Matsuo, Y., W-geometries, Phys. Lett. B274 (1992), 309-316.

8. Gervais, J.-L. and Matsuo, Y., Classical $A_{n}-W-$ Geometry, Commun. Math. Phys. 152 (1993), 317-368.

9. Gervais, J.-L. and Saveliex, M. V., W-Geometry of the Toda Systems Associated with Non-Exceptional Simple Lie Algebras, to appear in Commun. Math. Phys.

10. Givental', A. B., Plücker formulae and Cartan matrices, Russian Math. Surveys 44:3 (1989), 193-194.

11. Gorbatsevich, V. V., Onishchik, A. L. and Vinberg, E. B., Lie Groups and Lie Algebras, 3, Encyclopaedia of Math. Sciences, Vol. 41, New York, 1994.

12. Goto, M. and Grosshans, F., Semisimple Lie Algebras, Marcel Dekker, New York, 1978.

13. Griffiths, P., Periods of integrals on algebraic manifolds III, Publ. Math. I.H.E.S. 38 (1970), 125-180. 
14. Griffiths, P. and Harris, J., Principles of algebraic geometry, Interscience, New York, 1978.

15. Helgason, S., Differential geometry and symmetric spaces, Academic Press, New York, 1962.

16. Infinite dimensional Lie groups with applications (V. Kač, ed.), Springer, Berlin, 1985.

17. Kobayashi, S. and Nomizu, K., Foundations of differential geometry, Vols. 1, 2, Interscience, New York, 1963, 1969.

18. Leznov, A. N. and Saveliev, M. V., Group-theoretical methods for integration of nonlinear dynamical systems, Progress in Physics, v. 15, Birkhaüser-Verlag, Basel, 1992.

19. Leznov, A. N. and Shabat, A. B., Conditions of perturbation series breaking, Integrable systems (A. B. Shabat, ed.), pp. 34-44, Bashkirian Branch of the USSR Acad. of Science, Ufa, 1982 (in Russian).

20. Leznov, A. N., Smirnov, V. G. and Shabat, A. B., Internal symmetry group and integrability conditions for two-dimensional dynamical systems, Teor. Mat. Fiz. 51 (1982), 10-21.

21. Olive, D. I., Saveliev, M. V. and Underwood, J. W. R., On a solitonic specialisation for the general solutions of some two-dimensional completely integrable systems, Phys. Lett. B311 (1993), 117-122.

22. Positsel'skii, L. E., Local Plücker formulas for a semisimple Lie group, Funct. Anal. and its Appl. 25 (1991), 291-292.

23. Saveliev, M. V. and Savelieva, S. A., $W_{\infty}$-geometry and associated continuous Toda system, Phys. Lett. B313 (1993), 55-58.

24. Simpson, C., Higgs bundles and local systems, Publ. Math. I.H.E.S. 75 (1992), 5-95.

25. Yang, K., Almost complex homogeneous spaces and their submanifolds, World Scientific, Singapore, 1987.

Institute for High ENergy Physics, 142284 Protvino, Moscow Region, Russia.

E-mail address: razumov@m10.ihep.su

Laboratoire de Physique Théorique de l'Ecole Normale Supérieure ${ }^{1}, 24$ Rue Lhomond; 75231 PARIS CÉDEX 05, France (ON LEAVE of ABSENCE From Institute For High Energy Physics, 142284 Protvino, Moscow Region, Russia).

E-mail address: saveliev@physique.ens.fr

Received July 8, 1994. Revised September 8, 1994.

${ }^{1}$ Unité Propre du Centre National de la Recherche Scientifique, associée à l'Ecole Normale Supérieure et à l'Université de Paris-Sud. 